\title{
Area-Level Walkability and the Geographic Distribution of High Body Mass in Sydney, Australia: A Spatial Analysis Using the 45 and Up Study
}

\author{
Darren J. Mayne ${ }^{1,2,3,4, *}$, , Geoffrey G. Morgan ${ }^{1,5}$, Bin B. Jalaludin ${ }^{6,7}$ and Adrian E. Bauman ${ }^{1}$ \\ 1 The University of Sydney, School of Public Health, Sydney, NSW 2006, Australia; \\ geoffrey.morgan@sydney.edu.au (G.G.M.); adrian.bauman@sydney.edu.au (A.E.B.) \\ 2 Illawarra Shoalhaven Local Health District, Public Health Unit, Warrawong, NSW 2502, Australia \\ 3 University of Wollongong, School of Medicine, Wollongong, NSW 2522, Australia \\ 4 Illawarra Health and Medical Research Institute, University of Wollongong, \\ Wollongong, NSW 2522, Australia \\ 5 The University of Sydney, University Centre for Rural Health, Rural Clinical School-Northern Rivers, \\ Sydney, NSW 2006, Australia \\ 6 Ingham Institute, University of New South Wales, Sydney, NSW 2052, Australia; b.jalaludin@unsw.edu.au \\ 7 Epidemiology, Healthy People and Places Unit, Population Health, \\ South Western Sydney Local Health District, Liverpool, NSW 1871, Australia \\ * Correspondence: dmay8519@uni.sydney.edu.au; Tel.: +61-2-4221-6733
}

Received: 16 December 2018; Accepted: 19 February 2019; Published: 24 February 2019

check for updates

\begin{abstract}
Improving the walkability of built environments to promote healthy lifestyles and reduce high body mass is increasingly considered in regional development plans. Walkability indexes have the potential to inform, benchmark and monitor these plans if they are associated with variation in body mass outcomes at spatial scales used for health and urban planning. We assessed relationships between area-level walkability and prevalence and geographic variation in overweight and obesity using an Australian population-based cohort comprising 92,157 Sydney respondents to the 45 and Up Study baseline survey between January 2006 and April 2009. Individual-level data on overweight and obesity were aggregated to 2006 Australian postal areas and analysed as a function of area-level Sydney Walkability Index quartiles using conditional auto regression spatial models adjusted for demographic, social, economic, health and socioeconomic factors. Both overweight and obesity were highly clustered with higher-than-expected prevalence concentrated in the urban sprawl region of western Sydney, and lower-than-expected prevalence in central and eastern Sydney. In fully adjusted spatial models, prevalence of overweight and obesity was $6 \%$ and $11 \%$ lower in medium-high versus low, and $10 \%$ and $15 \%$ lower in high versus low walkability postcodes, respectively. Postal area walkability explained approximately $20 \%$ and $9 \%$ of the excess spatial variation in overweight and obesity that remained after accounting for other individual- and area-level factors. These findings provide support for the potential of area-level walkability indexes to inform, benchmark and monitor regional plans aimed at targeted approaches to reducing population-levels of high body mass through environmental interventions. Future research should consider potential confounding due to neighbourhood self-selection on area-level walkability relations.
\end{abstract}

Keywords: body mass; disease mapping; geographic variation; obese; overweight; spatial analysis; walkability 


\section{Introduction}

The increasing prevalence of overweight and obesity is a universal and urgent public health problem [1]. High body mass index $\geq 25 \mathrm{~kg} / \mathrm{m}^{2}$ (overweight or obese) contributed $5.7 \%$ of total disability adjusted life years (DALY) to the global burden of disease in 2016, making it the fifth leading risk factor-up from $2.7 \%$ of total DALYs and a ranking of 12 in 1990 [2]. High body mass is a risk factor for cardiovascular disease, cancer, type 2 diabetes mellitus, and musculoskeletal conditions [3,4], while its economic costs to health care systems and communities grow with increasing levels of overweight and obesity [5]. The physiological energy imbalance that underlies high body mass is influenced by genetic, behavioural, social, economic, and environmental factors operating within multiple complex systems [6-8]. Reducing the health and economic burdens of overweight and obesity will require shifts in these population-level systems [7]. For example, environmental interventions that typically produce small individual-level effects may aggregate into large population-level benefits because exposure is ubiquitous [8,9] and relatively persistent $[10,11]$.

The built environment refers to that "part of the physical environment...Constructed by human activity" ( [12] p. S550), and is hypothesised to contribute to high body mass by influencing lifestyle behaviours that underlie its development [8]. The emerging consensus from the extensive literature is that the built environment evidence base is sufficiently developed to incorporate into planning, policy and interventions aimed at reducing high body mass $[7,13]$, although uncertainties remain (see reviews by [14-18]). "Walkability" describes the capacity of the built environment to promote walking for multiple purposes [19], and may contribute to reducing overweight and obesity by promoting participation in total daily moderate-intensity physical activity $[8,9,17,20-22]$. To this end, it is increasingly considered in development plans aimed at enhancing physical and social infrastructure to promote healthy lifestyles and reduce the burden of chronic conditions like high body mass on populations (e.g., [23-26]).

Walkability indexes have been identified as potentially useful tools for planning, benchmarking and monitoring environmental policies and interventions to improve walkability, and translating the outcomes of walkability research from rhetoric to action $[27,28]$. While numerous indexes exist (e.g., [29-37]), the Neighborhood Quality of Life Study (NQLS) [38] and Physical Activity in Localities and Community Environments (PLACE) Study [19] indexes remain the most influential [39]; underpin a majority of research linking walkability to health behaviours and outcomes, including high body mass [17]; and are applicable in planning, policy and practice settings [28], which is facilitated by their capacity to be constructed at multiple spatial resolutions [38,40]. These indexes operationalise walkability using residential density, street connectivity, land use mix and, if available, retail floor area ratio, destinations or density within a geographic information system $[19,38,40]$. Index variables serve as proxy measures for built environment attributes associated with walking. Land use mix measures the diversity and concentration of land uses in an area, while intersection density measures the directness of paths [38]. Compact areas with diverse land uses that are highly connected promote walking by reducing the distance between origins and destinations $[9,19,40]$. Similarly, high population densities provide critical masses that concentrate diverse destinations within compact areas $[12,38,40]$, and is measured by residential density. The ratio of retail floor area to retail land use is a measure of pedestrian access, with larger values indicating greater area given to pedestrian uses and less area to car parking [41].

The extent to which walkability indexes are associated with health outcomes at population scales is a key consideration in their utility to benchmark and guide planning and policy aimed at reducing population-levels of overweight and obesity [27,42]. This is because health and urban planning that influences environmental walkability occurs at local, urban and regional scales $[43,44]$; that is, for communities and populations. These meso (neighbourhoods/communities) and macro (cities) geographic scales are much coarser than typically used in studies to derive built environment exposure-response evidence [42,45], which mostly focus on individual (micro) level risk (see reviews by $[14-17,22,46]$ ). Measured at the micro-scale, walkability is typically derived 
within a radial or street-based network buffer of 200-1600 metres around a residential address; reflects the immediate built environment to which an individual is exposed [47,48]; and is preferred for individual-level research.

In contrast, when measured at meso- and macro-scales, walkability (or sprawl) is usually calculated within an administrative boundary; represents a contextual variable describing the shared built environment to which groups, communities and populations are exposed; and is especially useful for area-level (ecological) research and planning applications [42]. Using individual-level walkability evidence to inform activity at coarser planning scales has raised concern in the literature for its potential to result in flawed public health action [49]. This is a concern about atomistic [50] or individualistic [51] fallacy, which is the area-level corollary of the ecological fallacy and refers to the erroneous use of data on individuals to make inferences about groups [52].

Analysis at the geographic scale of planning addresses concern about erroneous cross-level inference [42], and has been identified as highly relevant to "local area" walkability planning because it produces evidence at the level where decisions are made [43]. Rydin and colleagues have also identified the need for "urban scale" data to inform planning and policy interventions that maintain the urban advantage in health outcomes [44]. However, studies that match walkability exposures and body mass outcomes at these planning scales are uncommon $[16,17,46]$, despite calls from planners and policy makers for evidence at this level [45,53-55]. What evidence is available at these planning scales comes largely from ecological analyses in the United States, which have consistently found higher body mass and prevalence of obesity in sprawling versus compact counties (e.g., [30,53,56-58]). Compactness at this scale is generally considered synonymous with more walkable environments. However, sprawl indexes have been criticized for conflating multiple built environment concepts, and not providing a coherent, unitary measure of walkability $[38,40]$.

Geographic variation in overweight and obesity has been reported within many countries [59], and needs to be considered in health programming and planning. In addition to identifying areas at increased risk of adverse health outcomes [59,60], geographic variation in excess of that due to known factors can indicate place-based influences on health that are distinct to the contextual effects arising from differences in the demographic, social and economic composition of populations between areas $[61,62]$. Spatial analysis is particularly useful in identifying these place-based effects because it quantifies the contribution of both observed and unobserved factors to geographic variation in outcomes while accounting for spatial autocorrelation that can lead to biased statistical inference [62]. In this context, geographic variation encompasses more than just differences between areas, which is well addressed in the body mass literature (e.g., [63-72]). It is also the spatial expression or distribution of this variation [73]. Spatial analysis is concerned with location [74]. It leverages the underlying process giving rise to the geographic variation rather than reducing it to a naïve dummy-coded comparison of areas in the case of fixed-effects analysis, or focusing on reductions in intraclass correlation coefficients that conflate spatial and non-spatial sources of variation through a common random effect term as in multilevel analysis [42].

Spatial analysis has the potential to provide unique information on relations between walkability and high body mass. For example, we have previously reported that physical activity is geographically structured in Sydney, and that area-level walkability accounts for some of this spatial patterning [42]. The "disease mapping" approach [75] used in this study also produces smoothed maps that can be used to communicate spatially varying risks to planners, policy-makers and other interested stakeholders [42]. Identifying spatial disparities in contextual factors that contribute to adverse health outcomes at appropriate intervention scales has been identified as essential for informing place-based interventions aimed at improving population health [76]. Spatial analysis is uniquely placed to assist in addressing these disparities and environmental inequalities through its capacity to identify and target geographic areas where environment-related health risks are disproportionately higher and potentially amenable to intervention [42,77]. However, despite an increasing use of geographic information systems in the high body mass literature, the application of spatial analysis at any scale 
is rare [78]. For example, only a few area-level studies have used an explicitly spatial approach to address geographic variation in overweight and obesity [56,59,62,72,79-89]; an even smaller number have considered built environment influences on this geographic variation [62,81,82,89]; and only one appears to have evaluated the contribution of walkability to this geographic variation directly [81].

The objective of this study was to build on our previous work in the Sydney statistical district $[39,42]$ and assess relations between area-level walkability and population-levels of overweight and obesity using an explicitly spatial approach, and at a geographic scale representative of those used for "local area" [43] and "urban scale" [44] planning. The specific aims of the study were to (i) assess area-level associations between walkability and prevalence of overweight and obesity in Sydney; (ii) assess geographic variation in area-level prevalence of overweight and obesity in Sydney; and (iii) assess the extent to which area-level walkability accounts for geographic variation in overweight and obesity in Sydney beyond that due to individual-level demographic, social, economic and health factors, and area-level socioeconomic disadvantage.

\section{Materials and Methods}

\subsection{Study Design and Area}

We investigated associations between area-level walkability and prevalence of overweight and obesity in the Sydney statistical division of New South Wales, Australia [90], using a cross-sectional ecological study design, which is appropriate and valid for area-level inference [52]. Sydney has a land area of 12,142 km², and was Australia's most populous city at the 2006 Australian Census with an estimated resident population of 4.1 million people living in 1.6 million dwellings [91]. We used Census postal areas as our units of analysis to coincide with the smallest spatial unit at which individual-level data were geographically identified by the data custodian. In 2006 there were 260 conterminous postal areas across the Sydney statistical division [92] with median and inter quartile range (IQR) values for land area of $7.6(\mathrm{IQR}=3.7-19.4) \mathrm{km}^{2}, 5304(\mathrm{IQR}=2694-8426)$ residential dwellings, and 13,090 $(\mathrm{IQR}=6529-22,092)$ residents [91]. The median land area of postal areas corresponds to a radial buffer of $1550 \mathrm{~m}$, which is within the range of buffer sizes for which consistent environment-behaviour associations have been reported in individual-level studies of walkability [47,48], and is likely a reasonable analogue of the "local areas" and "urban scales" at which health and urban planning decisions occur $[43,44]$.

\subsection{Participants}

Individual-level data used in this study were obtained from participants of The Sax Institute 45 and Up Study [93] approved and monitored by the University of New South Wales Human Research Ethics Committee (ref no. HREC 05035/HREC 10186). This population-based cohort study was established to investigate healthy ageing in the New South Wales population aged 45 years and over [93]. Study recruitment occurred between January 2006 and December 2009 [94] for a final cohort size of 267,153 participants or approximately $10 \%$ of the total New South Wales target population [95]. Eligible persons were randomly sampled from the the Department of Human Services (formerly Medicare Australia) enrolment database. Selected individuals were mailed an invitation letter, and asked to return a signed, written consent form with their baseline survey via reply-paid mail if they consented to participating in the study [93]. We were provided access to the April 2009 data release, which the data custodian had geocoded to 2006 Census statistical divisions and postal areas. We limited our analysis to 115,153 respondents living in the Sydney statistical division to coincide with the spatial extent of our exposure variable. Our research comprised a sub-study of the Social, Environmental, and Economic Factors Study approved and monitored by the University of Sydney Human Research Ethics Committee (ref No. 10-2009/12187). Details on accessing 45 and Up Study data are available on The Sax Institute website (www.saxinstitute.org.au/our-work/45-up-study). 


\subsection{Data}

Individual-level data included self-reported responses to the baseline survey of 45 and Up Study collected between January 2006 and April 2009 [93], which we used to calculate and adjust area-level outcome variables. Postal area contextual variables comprised the Sydney Walkability Index (SWI) [40] and 2006 Index of Relative Socioeconomic Disadvantage [96], which we included as study and covariate factors, respectively.

\subsection{Outcome Variable}

The primary outcome measures used in our study were self-reported overweight and obesity, which we defined using the standard body mass index (BMI) formula of weight in kilograms $(\mathrm{kg})$ over height in metres $(\mathrm{m})$ squared $\left(\mathrm{kg} / \mathrm{m}^{2}\right)$ and World Health Organisation (WHO) cut-points of $25.0-<30.0 \mathrm{~kg} / \mathrm{m}^{2}$ for overweight and $\geq 30.0 \mathrm{~kg} / \mathrm{m}^{2}$ for obesity [97]. Self-reported BMI has been validated against measured BMI as a generally appropriate method for quantifying body size in the 45 and Up Study cohort, although it is known to underestimate prevalence of obesity when classified using standard BMI categories [98]. Overweight and obesity status were represented as dichotomous (yes/no) variables for individual-level analyses, and as counts of overweight and obese respondents within postcodes in area-level analyses.

\subsection{Exposure Variable}

The exposure variable used for all analyses was postal area walkability, which we measured using the Sydney Walkability Index [40]. This three-factor index is derived using methods and data comparable to the PLACE and NQLS walkability index $[19,38]$. The Sydney Walkability Index is calculated within a geographical information system using three built environment variables: residential dwelling density (the number of residential dwellings per square kilometre of residential land use); intersection density (the number of intersections with three or more roads per square kilometre of total land area); and land use mix (the entropy of residential, commercial, industrial, recreational and other land uses). The Sydney Walkability Index was derived at the 2006 postal area level using 2007 spatial data to temporally align it with the midpoint of the of the 45 and Up Study baseline data collection.

Environmental variable values are divided into deciles, scored from 1 (lowest) to 10 (highest), summed to give a total score between 3-30, and then divided into quartiles corresponding to low, low-medium, medium-high and high walkability [40]. Environmental values increase monotonically within strata and have median values of 2.3, 13.4, 19.8 and 46 dwellings per hectare for residential density; 3.4, 46.1, 79.5 and 162.5 intersections per square kilometre for street network connectivity; and entropies of $0.005,0.033,0.056$, and 0.134 for land use mix (see [42]). The Sydney Walkability Index has predictive validity for utilitarian walking, is comparable to four-variable indexes in the research literature, and is associated with population-levels of moderate and vigorous physical activity $[40,42]$.

Walkability was entered as an index in our analysis for consistency with the interest expressed in the literature on using "walkability indexes" to benchmark, inform and monitor regional development plans [27,28], and because the non-parametric functions used in other studies [99-101] to model index components separately would have made our already computationally-intensive spatial analyses intractable.

\subsection{Covariates}

Individual- and area-level factors from the 45 and Up Study and substantive literature likely to contribute to, or confound, associations between walkability and body mass were included as covariates in our analysis (see [102-113]). Individual-level covariates included self-reported sex; five-year age group at baseline interview; language spoken at home; educational level; relationship status; employment status; health insurance type; level of psychosocial distress measured using the 
Kessler Psychological Distress Scale [114] (minor, moderate, high, very high [115-117]); smoking status; number of chronic conditions ever diagnosed and treated in the previous four weeks; and functional capacity, which was measured using the Medical Outcomes Study (MOS) 36-Item Short-Form Health Survey (SF-36) physical functioning scale $[118,119]$ and classified as none $(0$ to $<60)$, minor $(60$ to $<90)$, moderate $(90$ to $<100)$, and severe (100) [120]. Postal area socioeconomic disadvantage was measured using the Index of Relative Socio-economic Disadvantage from the 2006 Australian Census [96]. We did not include physical activity in our analysis because it likely mediates relations between the built environment and high body mass [8,14-17].

\subsection{Statistical Analysis}

The objective of our analysis was to assess relations between walkability and the prevalence and geographic distribution of overweight and obesity in the Sydney statistical district at a scale analogous to those at which health and urban planning decisions are made. This objective is appropriately addressed by an ecological (spatial) analysis because the targets of inference are areas, not individuals [52]. We have previously identified high levels of spatial autocorrelation in 45 and Up Study data that have both research and planning utility, and the potential to bias inference if not addressed in the analysis [42]. Multilevel models can account for spatial autocorrelation but typically conflate spatial and non-spatial variation through a common variance component [42]. We therefore explicitly modelled the underlying spatial and non-spatial sources of variation in our data using a relative risk implementation of the ecological Besag, York and Mollié (BYM) conditional auto regressive model.

The BYM is a fully Bayesian ecological spatial model fit to aggregate data, which is commonly used in epidemiology for "disease mapping" applications [75]. The goal of disease mapping is to recover a map displaying variation in the geographic distribution of risks for spatial units within a study area that is "smoothed" of extreme and unreliable estimates that can arise from differences between units in the sizes of their underlying populations [75]. This is achieved in the BYM model by decomposing map variation into an unstructured variance component that smooths risk estimates towards the global mean of the study area, and a spatially structured (geographic) variance component that smooths risk estimates towards the local mean of contiguous spatial units $[75,121]$. These components also indicate the extent to which map variation is due to structured (spatial) and unstructured (non-spatial) factors.

The BYM model can be extended to ecological regression problems by incorporating area-level covariates into its specification [75], but it cannot parsimoniously control for individual-level factors that may confound area-level effect estimates. We therefore used a two stage modelling strategy adopted by other researchers in the epidemiological literature whereby individual-level regression models are used to estimate expected cases for each outcome, which are then used as offset terms in area-level spatial analyses to adjust for the varying size and composition of populations between spatial units (see [39,42,122-124]).

In the first step, we estimated the predicted log odds $\left(l_{i j}\right)$ of overweight and obesity for individuals using conditional fixed-effects logistic regression models:

$$
\hat{l}_{i j}=\alpha+x_{i} \beta
$$

where $\hat{l}_{i j}$ is the predicted log odds of being either overweight or obese for the $i^{\text {th }}$ person in the $j^{t h}$ postal area, $\alpha$ is the model intercept, and $x_{i} \beta$ is an optional vector of individual-level covariates. We fit two models for each outcome: (1) an unadjusted null model with no covariates; and (2) an adjusted model including all individual-level covariates described previously. The log odds for individuals from each model were converted to a predicted probability using the inverse link function:

$$
\hat{Y}_{i j}=\frac{e^{l_{i j}}}{1+e^{l_{i j}}}
$$


We then summed these probabilities within each postal area to obtain the expected number of cases for each outcome based on (1) the prevalence in the study area from unadjusted logistic regression estimates; and (2) the underlying respondent structure of our sample from adjusted logistic regression estimates (see [39,42,122-124]). These expected case counts were used as offsets in the spatial Poisson regressions described in step 2, and are referred to as unadjusted and adjusted offsets, respectively.

In the second step, we used relative risk implementations of the BYM model with Poisson likelihoods to estimate prevalence ratios for postal areas relative to the study area [125]. The BYM model is a fully Bayesian spatial model fit to aggregate data that decomposes total variation into observed and unobserved sources [75,121] using:

$$
\log \left(\theta_{j}\right)=\alpha+x_{j} \beta+s_{j}+u_{j}+\log \left(e_{j}\right)
$$

where $\theta_{j}$ is the prevalence ratio for the $j^{t h}$ postal area; $\alpha$ is the prevalence ratio for the study area; $x_{j}$ and $\beta$ are vectors of observed area-level explanatory variables and associated regression parameters estimates; $s_{j}$ and $u_{j}$ are unobserved spatially structured and unstructured random effects; and $e_{j}$ is an offset term representing the expected number cases in the $j^{\text {th }}$ area. The unstructured variance $\left(u_{j}\right)$ is a normal independent and identically distributed residual, while the spatial variance $\left(s_{j}\right)$ is conditionally normally distributed on the mean prevalence of the surrounding $k$ contiguous postal areas [75]. Model offsets $\left(e_{j}\right)$ corresponded to those derived for postal areas in step one, and were either unadjusted or adjusted for individual-level factors.

The total count of overweight and obese respondents $\left(o_{j}\right)$ in each postal area served as the dependent variable in each model. We fit six BYM spatial regressions for each outcome: (1) a null model with unadjusted offsets; (2) a null model with adjusted offsets; (3) a covariate model with adjusted offsets and postal area walkability; (4) a covariate model with adjusted offsets and postal area socioeconomic disadvantage; (5) a covariate model with adjusted offsets and postal area walkability and socioeconomic disadvantage, and (6) an effect modification model with adjusted offsets, postal area walkability and socioeconomic disadvantage, and their interaction. A total of 10,000 draws from the posterior distributions of two Monte Carlo Markov Chains sampled every 250th iteration were used to obtain medians and $95 \%$ credible intervals for each model. Chain convergence was assessed using autocorrelation plots and the Gelman-Rubin diagnostic [126]. We chose between alternate models using the Deviance Information Criterion (DIC) [127], and mapped exponentiated linear predictors and variance estimates using quintiles to visualise geographic variation in risk of high body mass. The spatial fraction $(\rho)$ for each model was calculated from the marginal variances of the random effects, and used to index the proportion of residual variation due to unobserved spatial factors (i.e., $\sigma_{s}^{2} /\left[\sigma_{s}^{2}+\sigma_{u}^{2}\right]$ ) (see $[128,129]$ ). Models were fit in WinBUGS 1.4.3 using R 3.3.2 and unweighted survey data, which produce unbiased, representative and generalisable relative effect estimates for individual- and area-level analyses in this cohort [42,130,131].

\section{Results}

Complete data were available for 92,157 of 115,153 (80.0\%) Sydney respondents residing in 254 of $260(97.7 \%)$ study postal areas. The median number of respondents per postal area was 212 , and ranged from 0 to 2532 with an inter-quartile range of 110-363. Individual-level attributes for respondents included in analyses are shown in the Characteristics section of Table 1. Consistent with the larger 45 and Up Study cohort [132], our sample had a similar gender and employment profile to the study area but was otherwise younger, more highly educated, less likely to speak a language other than English at home, and more likely to be living with a partner than the general Sydney population aged 45 years and over [91]. 
Table 1. Sample characteristics and prevalence of overweight and obesity among study participants.

\begin{tabular}{|c|c|c|c|c|c|c|}
\hline \multirow{3}{*}{ Variable } & \multicolumn{2}{|c|}{ Characteristics } & \multicolumn{4}{|c|}{ Prevalence } \\
\hline & \multirow[b]{2}{*}{$\mathbf{N}$} & \multirow[b]{2}{*}{$\%$} & \multicolumn{2}{|c|}{ Overweight } & \multicolumn{2}{|c|}{ Obesity } \\
\hline & & & $\mathbf{n}$ & $\%$ & $\mathbf{n}$ & $\%$ \\
\hline \multicolumn{7}{|l|}{ AREA-LEVEL VARIABLES } \\
\hline \multicolumn{7}{|l|}{ Walkability } \\
\hline Low & 25,454 & 27.6 & 10,150 & 52.9 & 6251 & 40.8 \\
\hline Low-medium & 31,404 & 34.1 & 12,380 & 50.0 & 6655 & 35.0 \\
\hline Medium-high & 19,449 & 21.1 & 7543 & 47.2 & 3454 & 29.0 \\
\hline High & 15,850 & 17.2 & 5861 & 44.0 & 2516 & 25.2 \\
\hline \multicolumn{7}{|l|}{ Socioeconomic disadvantage } \\
\hline Q1 - Most & 17,425 & 18.9 & 6697 & 52.1 & 4559 & 42.5 \\
\hline Q2 & 19,517 & 21.2 & 7579 & 51.7 & 4847 & 40.6 \\
\hline Q3 - Middling & 14,984 & 16.3 & 5877 & 49.4 & 3082 & 33.8 \\
\hline Q4 & 19,982 & 21.7 & 7938 & 47.8 & 3392 & 28.2 \\
\hline Q5 - Least & 20,249 & 22.0 & 7843 & 45.5 & 2996 & 24.1 \\
\hline \multicolumn{7}{|c|}{ INDIVIDUAL-LEVEL VARIABLES } \\
\hline \multicolumn{7}{|l|}{ Sex } \\
\hline Male & 44,690 & 48.5 & 20,802 & 58.1 & 8912 & 37.3 \\
\hline Female & 47,467 & 51.5 & 15,132 & 40.3 & 9964 & 30.8 \\
\hline \multicolumn{7}{|l|}{ Age } \\
\hline $45-49$ & 13,550 & 14.7 & 4871 & 45.1 & 2761 & 31.8 \\
\hline $50-54$ & 16,723 & 18.1 & 6188 & 47.4 & 3665 & 34.8 \\
\hline $55-59$ & 16,717 & 18.1 & 6568 & 51.2 & 3885 & 38.3 \\
\hline $60-64$ & 13,742 & 14.9 & 5696 & 53.7 & 3136 & 39.0 \\
\hline $65-69$ & 10,188 & 11.1 & 4297 & 54.0 & 2227 & 37.8 \\
\hline $70-74$ & 6910 & 7.5 & 2969 & 53.3 & 1341 & 34.0 \\
\hline $75-79$ & 4999 & 5.4 & 2047 & 49.0 & 820 & 27.8 \\
\hline $80-84$ & 6614 & 7.2 & 2513 & 43.2 & 801 & 19.5 \\
\hline $85+$ & 2714 & 2.9 & 785 & 31.7 & 240 & 12.4 \\
\hline \multicolumn{7}{|l|}{ Language spoken at home } \\
\hline English & 78,028 & 84.7 & 30,768 & 49.9 & 16,330 & 34.6 \\
\hline Other & 14,129 & 15.3 & 5166 & 44.6 & 2546 & 28.4 \\
\hline \multicolumn{7}{|l|}{ Education level } \\
\hline Less than secondary school & 7434 & 8.1 & 2704 & 50.6 & 2086 & 44.1 \\
\hline Secondary school graduation & 26,741 & 29.0 & 10,171 & 49.2 & 6052 & 36.5 \\
\hline Trade, certificate or diploma & 28,932 & 31.4 & 11,814 & 51.8 & 6143 & 35.9 \\
\hline University degree & 29,050 & 31.5 & 11,245 & 46.0 & 4595 & 25.8 \\
\hline \multicolumn{7}{|l|}{ Relationship status } \\
\hline Partner & 68,759 & 74.6 & 27,826 & 50.7 & 13,863 & 33.9 \\
\hline No partner & 23,398 & 25.4 & 8108 & 44.1 & 5013 & 32.8 \\
\hline \multicolumn{7}{|l|}{ Employment status } \\
\hline Full-time work & 32,716 & 35.5 & 13,622 & 53.5 & 7246 & 37.9 \\
\hline Part-time work & 13,177 & 14.3 & 4418 & 41.0 & 2408 & 27.5 \\
\hline Other work & 1358 & 1.5 & 426 & 39.6 & 281 & 30.2 \\
\hline Not working & 44,906 & 48.7 & 17,468 & 48.6 & 8941 & 32.6 \\
\hline \multicolumn{7}{|l|}{ Health insurance type } \\
\hline Private with extras & 54,218 & 58.8 & 21,751 & 50.1 & 10,830 & 33.4 \\
\hline Private without extras & 12,961 & 14.1 & 5058 & 47.2 & 2255 & 28.5 \\
\hline Government health care card & 11,993 & 13.0 & 4351 & 47.8 & 2881 & 37.7 \\
\hline None & 12,985 & 14.1 & 4774 & 47.4 & 2910 & 35.4 \\
\hline \multicolumn{7}{|l|}{ Smoking status } \\
\hline Never smoked & 54,117 & 58.7 & 20,518 & 46.6 & 10,072 & 30.0 \\
\hline Past smoker & 31,639 & 34.3 & 13,145 & 54.2 & 7397 & 40.0 \\
\hline Current smoker & 6401 & 6.9 & 2271 & 45.5 & 1407 & 34.1 \\
\hline
\end{tabular}


Table 1. Cont.

\begin{tabular}{|c|c|c|c|c|c|c|}
\hline \multirow{3}{*}{ Variable } & \multicolumn{2}{|c|}{ Characteristics } & \multicolumn{4}{|c|}{ Prevalence } \\
\hline & \multirow[b]{2}{*}{$\mathbf{N}$} & \multirow[b]{2}{*}{$\%$} & \multicolumn{2}{|c|}{ Overweight } & \multicolumn{2}{|c|}{ Obesity } \\
\hline & & & $\mathrm{n}$ & $\%$ & $\mathbf{n}$ & $\%$ \\
\hline \multicolumn{7}{|c|}{ Psychosocial distress } \\
\hline Low & 70,218 & 76.2 & 27,960 & 49.1 & 13,318 & 31.5 \\
\hline Moderate & 14,573 & 15.8 & 5433 & 49.0 & 3475 & 38.0 \\
\hline High & 5152 & 5.6 & 1828 & 48.4 & 1375 & 41.4 \\
\hline Very high & 2214 & 2.4 & 713 & 47.3 & 708 & 47.2 \\
\hline \multicolumn{7}{|c|}{ Diagnosed chronic conditions } \\
\hline 0 & 31,297 & 34.0 & 11,955 & 44.1 & 4218 & 21.8 \\
\hline 1 & 36,917 & 40.1 & 14,726 & 50.2 & 7560 & 34.1 \\
\hline 2 & 18,186 & 19.7 & 7145 & 54.4 & 5040 & 45.6 \\
\hline 3 or more & 5757 & 6.2 & 2108 & 57.0 & 2058 & 56.4 \\
\hline \multicolumn{7}{|c|}{ Treated chronic conditions } \\
\hline 0 & 41,580 & 45.1 & 15,904 & 45.5 & 6590 & 25.7 \\
\hline 1 & 30,121 & 32.7 & 12,141 & 51.3 & 6448 & 35.9 \\
\hline 2 & 14,524 & 15.8 & 5721 & 53.5 & 3835 & 43.6 \\
\hline 3 or more & 5932 & 6.4 & 2168 & 55.2 & 2003 & 53.2 \\
\hline \multicolumn{7}{|c|}{ Limited physical functioning } \\
\hline None & 32,392 & 35.1 & 12,656 & 44.4 & 3908 & 19.8 \\
\hline Minor & 25,125 & 27.3 & 10,628 & 52.4 & 4838 & 33.4 \\
\hline Moderate & 20,316 & 22.0 & 7801 & 52.8 & 5555 & 44.4 \\
\hline Severe & 14,324 & 15.5 & 4849 & 49.7 & 4575 & 48.3 \\
\hline \multicolumn{7}{|c|}{ SENSITIVITY VARIABLES } \\
\hline \multicolumn{7}{|c|}{ Total physical activity } \\
\hline $0 \mathrm{~min}$ & 5478 & 5.9 & 1868 & 50.9 & 1807 & 50.1 \\
\hline $1-149 \mathrm{~min}$ & 15,365 & 16.7 & 5895 & 52.1 & 4053 & 42.8 \\
\hline $150-299 \mathrm{~min}$ & 15,833 & 17.2 & 6241 & 50.5 & 3468 & 36.2 \\
\hline$\geq 300 \mathrm{~min}$ & 55,481 & 60.2 & 21,930 & 47.7 & 9548 & 28.5 \\
\hline
\end{tabular}

\subsection{Prevalence Overweight and Obesity}

The within-cohort prevalence of overweight and obesity were $49.0 \%(48.7-49.4 \%)$ and $33.6 \%$ (33.2-34.0\%), respectively. Table 1 reports prevalence by area- and individual-level characteristics. Prevalence of both overweight and obesity were highest in postal areas with low walkability, lowest in postal areas with high walkability, and displayed a exposure-response gradient. Likewise, overweight and obesity reduced with reducing levels of postal area socioeconomic disadvantage. For individual-level factors, overweight and obesity were more prevalent in males, persons speaking English at home or living with a partner, less educated individuals and full-time workers, persons without private health insurance, and past smokers; and increased with age to 65-69 years, psychosocial distress, number of diagnosed and treated chronic health conditions, and reduced functional capacity.

\subsection{Individual-Level Factors}

Table 2 shows adjusted fixed-effects estimates for overweight and obesity used to derive adjusted offsets for postal area spatial models. All effects were significantly associated with body mass outcomes and mostly consistent with the prevalence patterns reported in Table 1. The stand-out exception was a reversal in gradient between obesity and psychosocial distress from positive to negative after adjustment. This was due to confounding by functional capacity, which was both an independent risk factor for obesity (see Table 2) and strongly associated with psychosocial distress $\left(\chi_{9}^{2}=4072.4, p<0.0001\right)$. Other notable differences following adjustment were relationship status, which was unrelated to either overweight or obesity; age, which became associated with monotonically 
decreasing odds of obesity across the lifespan; and smoking status, which became associated with reduced odds of obesity for current compared to non smokers.

Table 2. Adjusted odds ratios for individual-level analyses of overweight and obesity.

\begin{tabular}{|c|c|c|c|c|}
\hline & \multicolumn{2}{|c|}{ Overweight } & \multicolumn{2}{|c|}{ Obese } \\
\hline & OR & $95 \% \mathrm{CI}$ & OR & $95 \%$ CI \\
\hline Sex & \multicolumn{2}{|c|}{$p<0.0001$} & \multicolumn{2}{|c|}{$p<0.0001$} \\
\hline Male & 1.00 & & 1.00 & \\
\hline Female & 0.47 & $0.46-0.49$ & 0.62 & $0.59-0.64$ \\
\hline Age & \multicolumn{2}{|c|}{$p<0.0001$} & \multicolumn{2}{|c|}{$p<0.0001$} \\
\hline $45-49$ & 1.00 & & 1.00 & \\
\hline $50-54$ & 1.00 & $0.95-1.05$ & 0.94 & $0.88-1.00$ \\
\hline $55-59$ & 1.07 & $1.01-1.13$ & 0.90 & $0.84-0.97$ \\
\hline $60-64$ & 1.08 & $1.02-1.15$ & 0.76 & $0.70-0.82$ \\
\hline $65-69$ & 1.00 & $0.93-1.07$ & 0.59 & $0.54-0.65$ \\
\hline $70-74$ & 0.87 & $0.81-0.94$ & 0.39 & $0.35-0.43$ \\
\hline $75-79$ & 0.66 & $0.60-0.72$ & 0.23 & $0.21-0.26$ \\
\hline $80-84$ & 0.50 & $0.46-0.54$ & 0.12 & $0.11-0.14$ \\
\hline $85+$ & 0.31 & $0.28-0.35$ & 0.06 & $0.05-0.07$ \\
\hline Language spoken at home & \multicolumn{2}{|c|}{$p<0.0001$} & \multicolumn{2}{|c|}{$p<0.0001$} \\
\hline English & 1.00 & & 1.00 & \\
\hline Other & 0.81 & $0.78-0.84$ & 0.72 & $0.68-0.77$ \\
\hline Education level & \multicolumn{2}{|c|}{$p<0.0001$} & \multicolumn{2}{|c|}{$p<0.0001$} \\
\hline Less than secondary school & 1.53 & $1.43-1.63$ & 2.47 & $2.28-2.67$ \\
\hline Secondary school graduation & 1.35 & $1.29-1.40$ & 1.77 & $1.67-1.86$ \\
\hline Trade, certificate or diploma & 1.27 & $1.22-1.32$ & 1.54 & $1.46-1.62$ \\
\hline University degree & 1.00 & & 1.00 & \\
\hline Relationship status & \multicolumn{2}{|c|}{$p<0.0001$} & \multicolumn{2}{|c|}{$p=0.1285$} \\
\hline Partner & 1.00 & & 1.00 & \\
\hline No partner & 0.89 & $0.86-0.92$ & 0.96 & $0.92-1.01$ \\
\hline Employment status & \multicolumn{2}{|c|}{$p<0.0001$} & \multicolumn{2}{|c|}{$p<0.0001$} \\
\hline Full-time work & 1.00 & & 1.00 & \\
\hline Part-time work & 0.75 & $0.71-0.79$ & 0.61 & $0.57-0.65$ \\
\hline Other work & 0.72 & $0.64-0.82$ & 0.61 & $0.52-0.71$ \\
\hline Not working & 0.78 & $0.75-0.82$ & 0.66 & $0.62-0.70$ \\
\hline Health insurance type & \multicolumn{2}{|c|}{$p<0.0001$} & \multicolumn{2}{|c|}{$p<0.0001$} \\
\hline Private with extras & 1.00 & & 1.00 & \\
\hline Private without extras & 0.90 & $0.86-0.94$ & 0.83 & $0.78-0.88$ \\
\hline Government health care card & 0.94 & $0.89-0.99$ & 1.02 & $0.96-1.09$ \\
\hline None & 0.91 & $0.87-0.95$ & 0.99 & $0.93-1.05$ \\
\hline Smoking status & \multicolumn{2}{|c|}{$p<0.0001$} & \multicolumn{2}{|c|}{$p<0.0001$} \\
\hline Never smoked & 1.00 & & 1.00 & \\
\hline Past smoker & 1.17 & $1.13-1.21$ & 1.28 & $1.23-1.34$ \\
\hline Current smoker & 0.78 & $0.74-0.84$ & 0.73 & $0.68-0.79$ \\
\hline Psychosocial distress & \multicolumn{2}{|c|}{$p<0.0001$} & \multicolumn{2}{|c|}{$p<0.0001$} \\
\hline Low & 1.00 & & 1.00 & \\
\hline Moderate & 0.94 & $0.90-0.98$ & 0.91 & $0.86-0.96$ \\
\hline High & 0.88 & $0.82-0.95$ & 0.82 & $0.76-0.89$ \\
\hline Very high & 0.83 & $0.74-0.92$ & 0.88 & $0.78-1.00$ \\
\hline Diagnosed chronic conditions & \multicolumn{2}{|c|}{$p<0.0001$} & \multicolumn{2}{|c|}{$p<0.0001$} \\
\hline 0 & 1.00 & & 1.00 & \\
\hline 1 & 1.19 & $1.15-1.24$ & 1.58 & $1.51-1.66$ \\
\hline 2 & 1.35 & $1.29-1.42$ & 2.13 & $2.01-2.27$ \\
\hline 3 or more & 1.48 & $1.37-1.60$ & 2.69 & $2.46-2.93$ \\
\hline
\end{tabular}


Table 2. Cont.

\begin{tabular}{|c|c|c|c|c|}
\hline & \multicolumn{2}{|c|}{ Overweight } & \multicolumn{2}{|c|}{ Obese } \\
\hline & OR & $95 \% \mathbf{C I}$ & OR & $95 \% \mathrm{CI}$ \\
\hline Treated chronic conditions & \multicolumn{2}{|c|}{$p<0.0001$} & \multicolumn{2}{|c|}{$p<0.0001$} \\
\hline 0 & 1.00 & & 1.00 & \\
\hline 1 & 1.22 & $1.18-1.27$ & 1.47 & $1.40-1.54$ \\
\hline 2 & 1.38 & $1.31-1.45$ & 1.89 & $1.77-2.01$ \\
\hline 3 or more & 1.57 & $1.45-1.69$ & 2.48 & $2.27-2.71$ \\
\hline Limited physical functioning & \multicolumn{2}{|c|}{$p<0.0001$} & \multicolumn{2}{|c|}{$p<0.0001$} \\
\hline None & 1.00 & & 1.00 & \\
\hline Minor & 1.36 & $1.30-1.41$ & 2.10 & $1.99-2.21$ \\
\hline Moderate & 1.58 & $1.51-1.65$ & 3.77 & $3.56-4.00$ \\
\hline Severe & 1.61 & $1.52-1.70$ & 5.31 & $4.96-5.68$ \\
\hline
\end{tabular}

OR-Odds ratio, $\mathrm{CI}-$ Confidence interval.

\subsection{Spatial Analysis}

Tables 3 and 4 report parameter estimates and diagnostics for spatial regressions fit to overweight and obesity data. Smoothed prevalance ratios for postal areas from unadjusted null models ranged from $0.83-1.16$ for overweight and $0.46-1.68$ for obesity. Variation in risks between postal areas was principally due to unobserved spatial factors, with $>96 \%$ of residual variation attributed to the spatial variance component for both overweigtht and obesity (see spatial fractions for Model 1 in Tables 3 and 4). Adjusting for individual-level factors (Model 2) attenuated the ranges of smoothed prevalence ratios to $0.88-1.08$ for overweight and $0.63-1.23$ for obesity, but had little effect on the proportions of residual variation from spatial sources, which remained high at $>93 \%$ for both outcomes. Univariable parameter estimates for area-level associations including walkability and socioeconomic disadvantage are shown in the Model 3 and 4 columns of Tables 3 and 4. Risk ratios for walkability indicated consistent exposure gradients for both outcomes, with prevalence of overweight reduced by $4 \%$ and $9 \%$ and obesity by $8 \%$ and $11 \%$ in medium-high and high versus low walkability postal areas. Likewise, high body mass reduced monotonically with decreasing socioeconomic disadvantage. Overweight was $6 \%$ lower in the least versus most disadvantaged postal areas, and obesity was $11 \%$ and $9 \%$ lower in the least and second-to-least versus most disadvantaged postal areas. Fully-adjusted spatial regressions including individual- and area-level factors (Model 5) had the lowest DIC values and were the best fitting models for both outcomes (see DIC row in Tables 3 and 4). Prevalence ratios for socioeconomic disadvantage in these models were largely unaffected; however, gradients for area-level walkability strengthened with overweight $6 \%$ and $10 \%$ lower and obesity $11 \%$ and $15 \%$ lower in medium-high and high versus low walkability postcodes. These fully-adjusted spatial models also had the smallest amounts of residual spatial variation, with $67 \%$ of unexplained model variation attributed to unobserved spatial factors for overweight and $90 \%$ for obesity. Interaction analyses (Models 6) provided no evidence that the observed associations between walkability and overweight $\left(D I C_{M 6}-D I C_{M 5}=18.21\right)$ or obesity $\left(D I C_{M 6}-D I C_{M 5}=12.12\right)$ were modified by postal area socioeconomic disadvantage. 
Table 3. Spatial regression summaries for postal area analyses of associations between overweight, walkability and relative socioeconomic disadvantage.

\begin{tabular}{|c|c|c|c|c|c|}
\hline & Model 1 & Model 2 & Model 3 & Model 4 & Model 5 \\
\hline Individual-level adjustment & No & Yes & Yes & Yes & Yes \\
\hline \multicolumn{6}{|l|}{ Prevalence ratios (95\% CrI) } \\
\hline Constant & $0.99(0.98-1.00)$ & $1.00(0.98-1.01)$ & $1.03(1.00-1.06)$ & $1.01(0.99-1.04)$ & $1.07(1.02-1.11)$ \\
\hline \multicolumn{6}{|l|}{ Walkability } \\
\hline Low & - & - & 1.00 & - & 1.00 \\
\hline Low-medium & - & - & $0.98(0.95-1.01)$ & - & $0.98(0.95-1.01)$ \\
\hline Medium-high & - & - & $0.96(0.92-1.00)$ & - & $0.94(0.91-0.98)$ \\
\hline High & - & - & $0.91(0.87-0.97)$ & - & $0.90(0.86-0.94)$ \\
\hline \multicolumn{6}{|c|}{ Socioeconomic disadvantage } \\
\hline Q1 - Most & - & - & - & 1.00 & 1.00 \\
\hline$\widehat{\mathrm{Q}} 2$ & - & - & - & $1.01(0.97-1.05)$ & $1.01(0.97-1.04)$ \\
\hline Q3 - Middling & - & - & - & $0.99(0.95-1.03)$ & $0.99(0.95-1.03)$ \\
\hline Q4 & - & - & - & $0.97(0.93-1.01)$ & $0.97(0.93-1.00)$ \\
\hline Q5 - Least & - & - & - & $0.94(0.90-0.99)$ & $0.93(0.89-0.97)$ \\
\hline \multicolumn{6}{|l|}{ Model diagnostics } \\
\hline $\mathrm{pD}$ & 55.73 & 37.48 & 33.64 & 35.05 & 27.01 \\
\hline DIC & 1832.77 & 1787.67 & 1787.12 & 1787.85 & 1782.70 \\
\hline Spatial fraction & 0.965 & 0.932 & 0.882 & 0.900 & 0.673 \\
\hline
\end{tabular}

CrI-credible interval, pD—effective parameters, DIC—Deviance Information Criterion. Model 1—null model with expected cases proportional to the overall prevalence. Model 2 -null model with expected cases adjusted for individual-level factors. Model 3-Model 2 + Sydney Walkability Index. Model 4-Model 2 + Index of Relative Socioeconomic Disadvantage. Model 5-Model 3 + Index of Relative Socioeconomic Disadvantage. 
Table 4. Spatial regression summaries for postal area analyses of associations between obesity, walkability and relative socioeconomic disadvantage.

\begin{tabular}{|c|c|c|c|c|c|}
\hline & Model 1 & Model 2 & Model 3 & Model 4 & Model 5 \\
\hline Individual-level adjustment & No & Yes & Yes & Yes & Yes \\
\hline \multicolumn{6}{|l|}{ Prevalence ratios (95\% CrI) } \\
\hline Constant & $0.95(0.93-0.97)$ & $0.96(0.95-0.98)$ & $1.02(0.97-1.08)$ & $1.01(0.96-1.05)$ & $1.10(1.02-1.17)$ \\
\hline \multicolumn{6}{|l|}{ Walkability } \\
\hline Low & - & - & 1.00 & - & 1.00 \\
\hline Low-medium & - & - & $0.97(0.91-1.02)$ & - & $0.96(0.91-1.01)$ \\
\hline Medium-high & - & - & $0.92(0.85-0.99)$ & - & $0.89(0.83-0.96)$ \\
\hline High & - & - & $0.89(0.80-0.99)$ & - & $0.85(0.78-0.94)$ \\
\hline \multicolumn{6}{|c|}{ Socioeconomic disadvantage } \\
\hline Q1 - Most & - & - & - & 1.00 & 1.00 \\
\hline Q2 & - & - & - & $1.03(0.98-1.09)$ & $1.02(0.97-1.08)$ \\
\hline Q3 - Middling & - & - & - & $0.97(0.92-1.03)$ & $0.97(0.91-1.03)$ \\
\hline Q4 & - & - & - & $0.91(0.85-0.97)$ & $0.90(0.85-0.96)$ \\
\hline Q5 - Least & - & - & - & $0.88(0.82-0.95)$ & $0.85(0.79-0.92)$ \\
\hline \multicolumn{6}{|l|}{ Model diagnostics } \\
\hline $\mathrm{pD}$ & 128.60 & 72.36 & 70.99 & 63.02 & 56.79 \\
\hline DIC & 1794.88 & 1711.26 & 1712.90 & 1705.26 & 1703.00 \\
\hline Spatial fraction & 0.992 & 0.985 & 0.981 & 0.978 & 0.961 \\
\hline
\end{tabular}

CrI-credible interval, pD—effective parameters, DIC—Deviance Information Criterion. Model 1—null model with expected cases proportional to the overall prevalence. Model 1—-null model with expected cases proportional to the overall prevalence. Model 3-Model 2 + Sydney Walkability Index. Model 4-Model $2+$ Index of Relative Socioeconomic Disadvantage. Model 5-Model $3+$ Index of Relative Socioeconomic Disadvantage. 


\subsection{Prevalence Maps}

Figures 1 and 2 graphically display smoothed prevalence ratios for overweight and obesity obtained from spatial models 1 (unadjusted null model), 2 (adjusted null model) and 5 (adjusted model with walkability and socioeconomic disadvantage). Total excess prevalence is shown in Maps A, D and $\mathrm{G}$, and decomposed into excess risk due to spatial factors in maps $\mathrm{B}, \mathrm{E}$ and $\mathrm{H}$, and unstructured factors in maps C, F and I. Two features stand-out in each set of maps. First, residual prevalence is principally due to unobserved place-based factors, with higher ratios in spatial $(\mathrm{B}, \mathrm{E}$ and $\mathrm{H})$ versus unstructured (C, F and I) maps; and second, this geographic variation in risk reduces as individual- (Model 2) and area-level (Model 5) factors are added to spatial models. In unadjusted (Model 1) and adjusted (Model 2) null models, higher-than-expected prevalence was concentrated in western Sydney, and lower-than-expected prevalence in central and eastern Sydney. Including area-level walkability and relative socioeconomic disadvantage (Model 5) substantially attenuated excess prevalence by reducing excess risk attributable to unobserved spatial factors (see maps $G$ and $H$ of Figures 1 and 2). Final excess prevalence estimates were reduced in western Sydney and the peri-urban fringe for both overweight and obesity; and remained higher-than-expected for obesity through south-central Sydney, and lower-than-expected for both outcomes on the eastern seaboard north of the Sydney central business district.
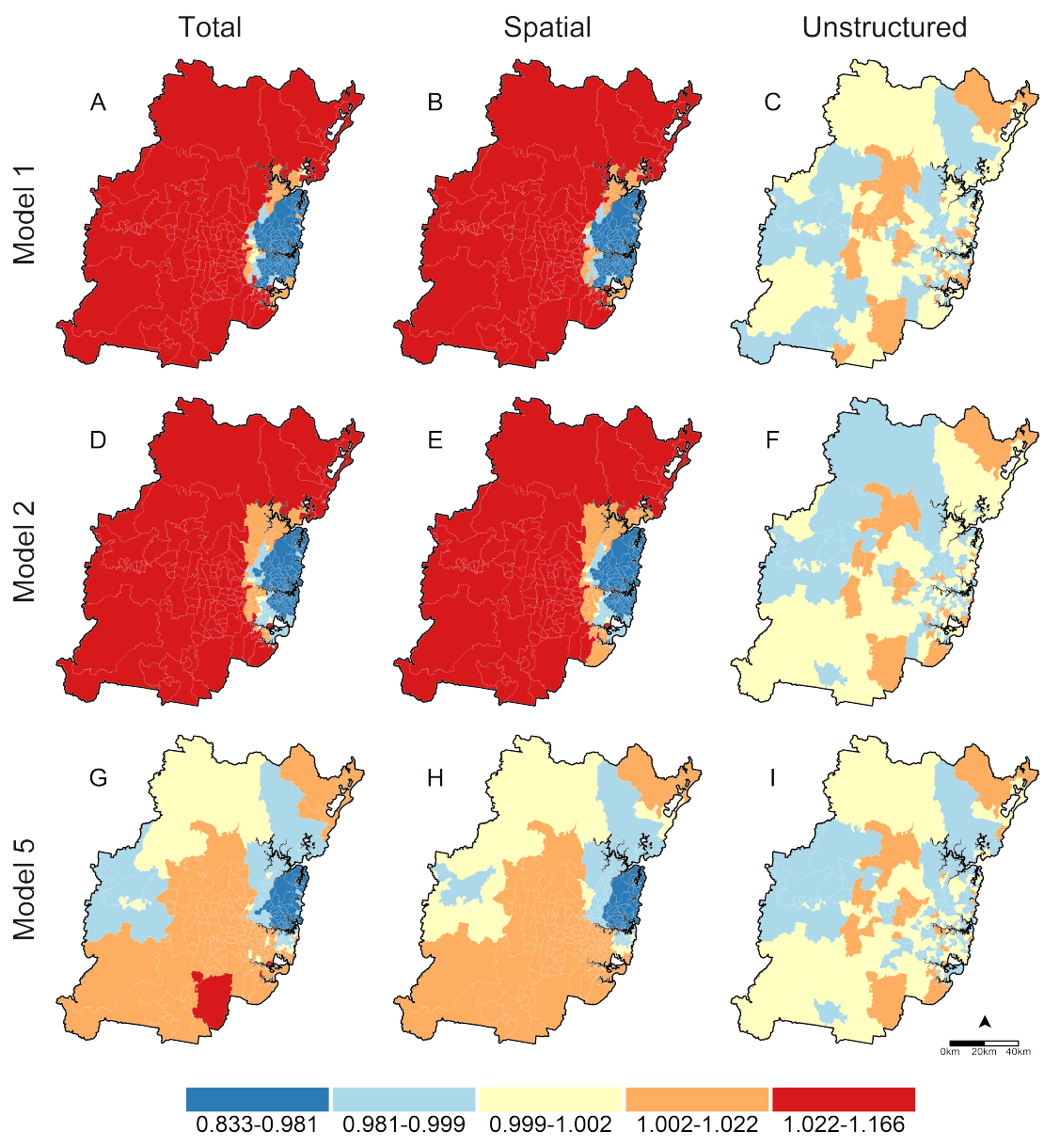

Figure 1. Total, Spatial and Unstructured prevalence ratios for overweight body mass in Sydney postal areas. Total prevalence ratios are derived by exponentiating the sum of spatial $(s)$ and unstructured $(u)$ random effects; Spatial and Unstructured prevalence ratios are obtained by exponentiating individual $s$ and $u$ components, respectively. Total, Spatial, and Unstructured prevalence ratio estimates are reported in maps (A-C) for Model 1, maps (D-F) for Model 2, and maps (G-I) for Model 5. 

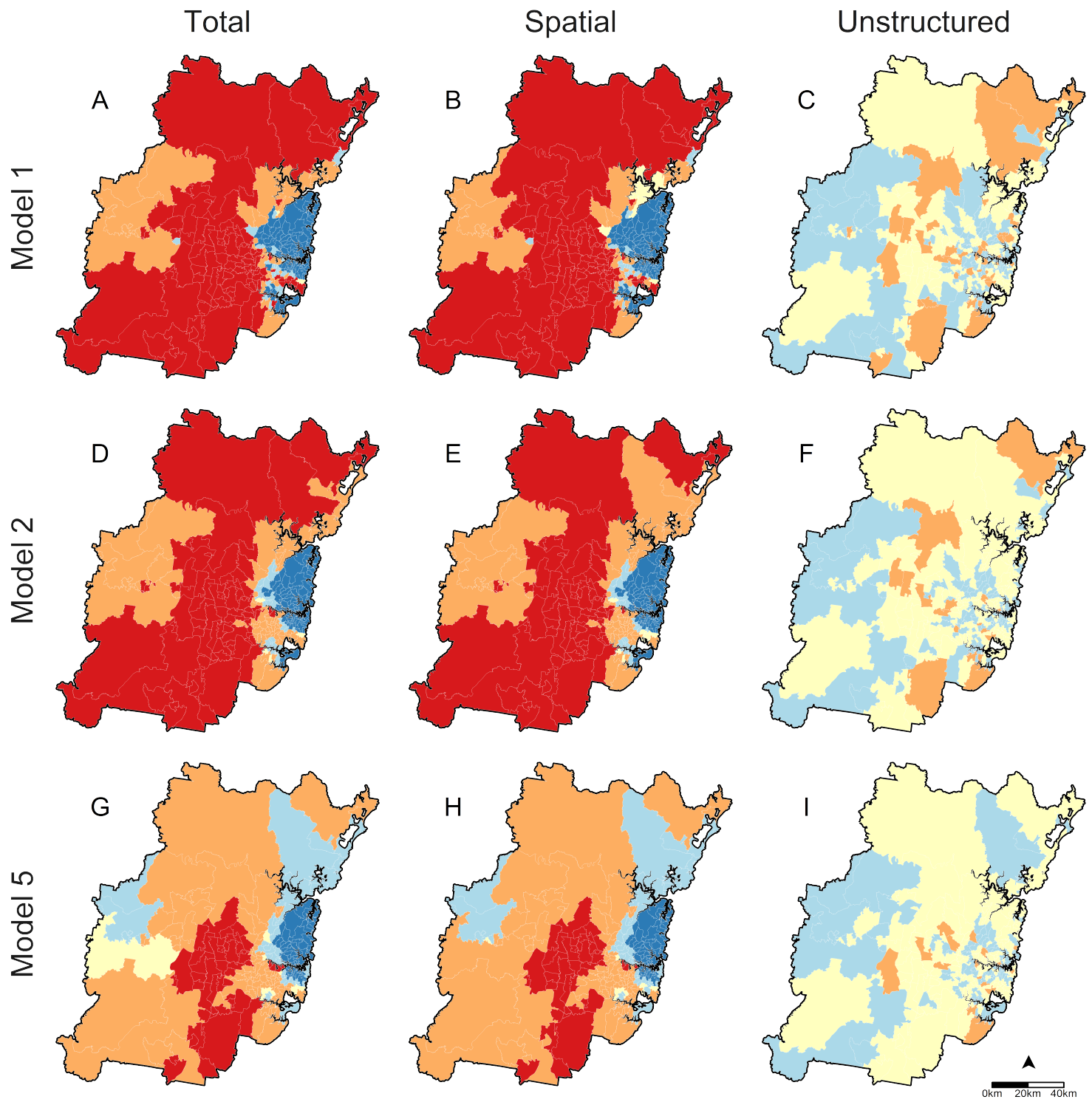

$$
\begin{array}{lllll}
0.478-0.935 & 0.935-0.999 & 0.999-1.005 & 1.005-1.095 & 1.095-1.764
\end{array}
$$

Figure 2. Total, Spatial and Unstructured prevalence ratios for obese body mass in Sydney postal areas. Total prevalence ratios are derived by exponentiating the sum of spatial $(s)$ and unstructured $(u)$ random effects; Spatial and Unstructured prevalence ratios are obtained by exponentiating individual $s$ and $u$ components, respectively. Total, Spatial, and Unstructured prevalence ratio estimates are reported in maps (A-C) for Model 1, maps (D-F) for Model 2, and maps (G-I) for Model 5.

\section{Discussion}

This is one of only a small number of studies to examine geographic variation in high body mass and its association with environmental walkability using a large population-derived cohort and spatial analytic framework. We find strong support for associations between postal area walkability and area-levels of overweight and obesity among persons aged 45 years and over living in Sydney, Australia. Prevalence in postal areas with medium-high and high walkability is reduced by $6 \%$ and $10 \%$ for overweight and $11 \%$ and $15 \%$ for obesity compared to postal areas with low walkability, and are independent of individual-level social, economic and health status factors, and area-level socioeconomic disadvantage. We also find that both overweight and obesity are geographically clustered at the postal area level with lowest prevalence in and to the north of the central business 
district, and highest prevalence in western Sydney. Postal area walkability explains approximately 20\% and $9 \%$ of this geographic variation in overweight and obesity, respectively, that is not attributable to individual-level factors and area-level socioeconomic disadvantage. Our findings confirm associations between high body mass and walkability at spatial scales typical of those used for public health planning; highlight the potential for spatial analysis to better integrate "place" into walkability research; and provide novel methods and data for New South Wales Government initiatives aimed at creating built environments that support active transportation and promote healthy lifestyles, and monitoring these initiatives.

Despite some limitations, the existing built environment evidence base appears sufficiently developed to inform interventions aimed at addressing high body mass at individual and population levels [13]. A recent review concluded that the strongest evidence is for meso- and macro-level correlates, and identifies urban sprawl, land use mix, street connectivity, population density, and proximity to services and destinations as the important environmental characteristics at these levels [17]. Walkability indexes combine many of these key environmental variables into summary metrics that can be easily implemented at multiple spatial scales for planning purposes $[19,27,28,40]$. Our study is novel because it directly addresses exposure-outcome relations at a geographic scale more proximal to those typically used by government agencies for population-level health and urban planning [43-45]. We observed that higher levels of postal area walkability measured by our index were associated with lower prevalence of overweight and obesity in postal area populations aged $\geq 45$ years, even after adjusting for other individual- and area-level characteristics related to body mass. These findings coincide with the small but consistent body of area-level findings that increased body mass and prevalence of obesity are associated with greater urban sprawl (see review by [17]), and extend recent individual-level associations between walkability and body mass [133-137] to populations and the spatial scales at which health and urban planning decisions are made. Our study also provides new evidence on the potential of tools like the Sydney Walkability Index [40] to benchmark, inform and monitor health and urban planning activities aimed at reducing population-levels of overweight and obesity. This will have relevance in the Australian context where open-access tools have been developed that allow researchers and planners to calculate NQLS-PLACE index values at mutiple spatial scales (see $[28,138]$ ).

Action to address overweight and obesity should target populations of greatest need $[59,60]$. However, it is unlikely that at-risk groups will be uniformly distributed across an area such as the Sydney statistical district [88]. Spatial analysis is especially useful in this regard with its ability to identify geographic locations with higher (or lower) than expected rates of overweight and obesity, and whether this variation is explained by, or in addition to, factors known to influence the distribution of health, such as demographic and socioeconomic characteristics [61]. We observed very strong clustering of overweight and obesity through central Sydney that was due to unobserved and spatially structured factors, and which contributed the majority of excess risk. Including individual-level demographic, social, economic and health status factors in our analysis attenuated excess prevalence of high body mass and reduced spatial variance but had little effect on outcome clustering across the study region. This is consistent with Canadian findings that individual-level variables were important correlates of within-region variation but explained little between-area geographic variation [85]. In contrast, adding postal-area walkability and socioeconomic disadvantage to our models reduced area-level clustering of overweight and, to a lesser extent, obesity. However, our final maps remained weakly clustered. This residual variation could suggest the presence of other unobserved spatial factors structuring the residual prevalence of high body mass in our study area. Identifying these additional factors was beyond the aims of our study but may include greenspace, access to shops and services, aesthetics, the food environment, and proximity to public transport [7,8,13,62]. It is also possible that some of this residual variation is due to residual confounding of associations between walkability and high body mass by sociodemographic factors. For example, Frank and colleagues have reported that features of walkable neighbourhoods are associated with lower overweight in males 
but greater overweight in females without a degree, and with lower obesity in men with a degree but higher obesity in unemployed non-white men without a degree and white women without a degree [139]. Likewise, there is some evidence that higher body mass is negatively associated with features of walkable neighbourhoods in high socioeconomic communities, and positively associated in low socioeconomic minority communities [140]. Future spatial studies employing our approach should consider alternate adjustment techniques to account for this possibility; for example, by calculating offsets using a logistic machine learning classifier.

Our findings are consistent with a growing evidence base indicating geographic variation at multiple spatial scales in the distribution of overweight and obesity that have relevance for health, urban and transport planning [56,59,62,79-89], although only a few studies have investigated built environment correlates of this variation within a geospatial context $[62,81,82]$. While Shuurman and colleagues were unable to assess whether population density-included in most walkability indexes-patterned obesity in Metro Vancouver because obesity itself did not cluster in their study area [82], Congdon has reported that not only are obesity rates $13-20 \%$ higher in sprawling versus compact US counties - an effect size similar to that obtained for physical inactivity, but that adding environmental measures to spatial models of county-level obesity prevalence reduced unexplained spatial variation by $22 \%$. Lathey et al. have also examined associations between obesity rates and sprawl factors, including walkability, for census blocks in Maricopa County, Arizona [81]. They defined walkability as accessibility to places of social interaction, and found it was the strongest model predictor of being in a "high disease" obesity cluster with odds halved for the most versus least walkable census blocks [81]. Cluster membership was also associated with residential and commercial land use, and street connectivity, although effect sizes were very small [81]. Unfortunately, the focus on correlates of cluster membership reduces the analysis to a consideration of between-group differences, which is not especially informative geographically. Our study adds to the evidence base by its explicit focus on walkability and its contribution to geographical variation in high body mass at the spatial scales where health and urban planning decisions are made. We found effect sizes for walkability that were meaningful at population-scales [10], and sizable reductions in unexplained spatial variation comparable to other area-level spatial analyses [62].

Despite substantial reductions in unexplained variation due to spatially structured factors of 93.6\% for overweight and $89.1 \%$ for obese, we observed little impact on spatial fractions except for overweight model 5 , which reduced from $\geq 88.2 \%$ (models $1-4$ ) to $67.3 \%$. This is not surprising given the unstructured variance reduced by just $12.9 \%$ for overweight and $46.1 \%$ for obesity over the range of models fitted; with most of this decrease occurring between models 1 and 2 when we first adjusted for individual-level factors. Lunn et al. have noted that either the spatial (s) or unstructured (u) variance component will typically dominate the other in practical implementations of the Besag, York and Mollié model but will only be apparent once the posterior distributions of the components are examined [141]. A key strength of the Besag, York and Mollie model is its robustness to spatial and non spatial variation, and will produce unbiased parameter and variance estimates in the absence of either [142]. The large residual spatial fractions from our analyses also suggests the likely existence of additional geographically distributed factors related to overweight and obesity within in the Sydney Statistical Division.

The geographic variation observed in our data and reported in the substantive literature highlight the importance of appropriately controlling for spatial autocorrelation at analysis [39,42,61]. Spatial autocorrelation is problematic for standard regression methods that assume model residuals are independently and identically distributed (IDD), and its violation may result in biased inference [143]. Clustering is most typically handled by multilevel models that conflate unexplained spatial and non-spatial variation into a single random effect error term [42]. This approach addresses the issue of spatial autocorrelation; however, the potential value of the spatial variation for informing health planning is lost in the process. We have consistently identified variation in health risk-factors and outcomes in the Sydney region using 45 and Up Study data that indicate geographic areas with excess 
risk attributable to unobserved and spatially structured factors [39,42]. For example, we have reported variation in physical activity [42] and psychosocial distress [39] that indicates excess risk due to unobserved and spatially structured factors in addition to that attributable to observed individual-level factors, and postal area walkability and relative socioeconomic disadvantage. Pattenden and colleagues contend that outcome variation in excess of socioeconomic factors may indicate opportunities to address disparities in health status [61], while Fitzpatrick et al highlight its potential role in suggesting causal pathways [144]. We believe our approach is helpful because it not only locates inequalities in the geographic distribution of risk but also quantifies that attributable to known factors that may, or may not, be amenable to intervention, and to unknown factors requiring further investigation.

We observed statistically significant associations between most individual-level covariates and body mass outcomes in all fixed-effects models used to derive offset terms in spatial models. Consistent with our previous work on physical activity [42] and psychosocial distress [39] in this cohort, we observed strong positive associations between prevalence of high body mass and numbers of chronic care conditions ever diagnosed and recently treated, and even stronger associations with reduced functional capacity. These findings agree with previous reports on this cohort $[103,120]$ and in the broader literature [145-147]. High body mass is considered a "gateway" into non-communicable diseases [148], and possibly multi-morbidity [149-152] and reduced physical functioning [145,153,154], although reverse causality is plausible with multi-morbidity and reduced physical functioning leading to lower levels of physical activity and poorer dietary choices [155]. We also observed an inverse association between high body mass and lower levels of psychosocial distress after adjusting for functional capacity. This is consistent with previous findings of increased risk of psychosocial distress with greater functional limitations in this cohort $[39,156,157]$, and a strong contemporaneous effect of physical disability on depressive symptoms [158]. Depression and anxiety disorders are also known causes of weight loss in community-dwelling older adults [159], and may be influential on our findings as the Kessler Psychological Distress Scale [114] is specific for current anxiety and affective disorders in Australian community populations at the cut-points used in our study [160].

A major strength of our study is the large sample size drawn from the 45 and Up Study [93]. This high-quality, population-based cohort comprises approximately $10 \%$ of the Sydney statistical district aged 45 and over. While we make no claims to the external validity of our point-estimates beyond our sample, it is well established in the epidemiological literature that relative measures of effect are generalisable irrespective of representativeness and non response [161,162]. Methodological investigations of the 45 and Up Study cohort support this likelihood. Mealing et al. [130] have reported that odds ratios derived from the full cohort correspond to those from the population-representative New South Wales Adult Population Health Survey [163], while we have reported high correlations between postal area relative risks and disease maps estimated from unweighted and post-stratification weighted data [42,131]. These observations support the generalisability of our risk estimators and their geographical distribution to postal area populations within the Sydney Statistical Division area. We also used the Sydney Walkability Index as our exposure metric, which is derived using high-quality government agency spatial data [40]. The strengths of this index include its demonstrated predictive validity for moderate-intensity walking at multiple spatial scales, a cohesive latent variable structure, and comparability to other indexes (e.g., NQLS [38] and PLACE [19]) frequently used in walkability research $[40,42]$. The spatial data used in its construction are routinely updated to support NSW Government business, and are accessible via the NSW Open Data Policy [164]. This allows the Sydney Walkability Index to be re-calculated annually to monitor changes in the spatial distribution of walkability across the Sydney statistical district. There is also an ongoing effort to develop a national walkability index using similar methods to our index that would benchmark and monitor changes in walkability across Australia [28]. Finally, our study employed an explicitly spatial approach that controlled for individual-level factors to investigate geographic variation in high body mass and its association with environmental walkability at the postal area level. The substantial levels of clustering in our data indicate the importance of accounting for spatial autocorrelation in analyses where it is 
expected or observed, and highlights the potentially informative nature of this variation for health and urban planning that is ignored when spatial and non-spatial sources of variation are conflated [42].

Our study reported on associations between postal area walkability and high body mass outcomes, which are not necessarily causal. An important limitation of our study is that we were unable to control for potential bias due to participant self-selection into postal areas, which raises the potential for reverse causation. Self-selection bias occurs when individuals choose to live in neighbourhoods that support their physical activity and travel behaviour preferences $[8,17]$. Systematic reviews indicate that neighbourhood self-selection may account for up to $50 \%$ of the built environment's effect on physical activity [17]. Its contribution to built environment associations with high body mass is less clear. Some studies have reported that self-selection fully accounts for these associations [165,166], while others have reported more modest attenuation effects [167-169]. There is also some evidence that it may selectively attenuate associations for continuous but not categorical body mass outcomes $[170,171]$. The 45 and Up Study does not collect information on respondents' preferences for the neighbourhoods in which they reside, and so we are unable to discount this as contributing to some portion of the estimated effect of walkability in our study.

We used self-reported BMI to classify overweight and obesity, which is generally appropriate for quantifying body size in the 45 and Up Study cohort but known to underestimate prevalence of obesity using standard BMI classifications by $6 \%$ [98]. In the context of our study, this means both overweight and obesity are likely to have been systematically misclassified. Monte Carlo simulation studies have found that systematic misclassification of binary dependent variables on the order of $2-5 \%$ can bias relative effects estimates by $12-25 \%$ in either direction [172,173]. This has the potential to weaken the magnitude of our observed associations for both overweight and obesity, but would still result in meaningful effect sizes at the population-level $[8,10]$. Another limitation of our analysis is that it was conducted at a single geographic scale, and so our findings may differ if conducted at a finer or coarser scale. This is the Modifiable Areal Unit Problem [174,175], which is germane to all analyses using areal units or zones [175]. We were only provided with access to postal area identifiers by the data custodian, and so were unable to assess the sensitivity of our results to different spatial scales. We have previously examined associations between walkability and health-enhancing physical activity at different spatial scales and found similar relations [40,42], which provides some reassurance on the robustness of our findings to spatial scale. However, the influence of scale on matched exposure-response relations in the walkability literature remains opens and warrants further investigation.

The Sydney Walkability Index [40] is comparable to other indexes used in the substantive literature [19] and so is subject to the same limitation that walkability quartiles may be data-dependent as they are derived using population-specific cut-points [176,177]. We therefore encourage planners, policy makers and researchers to review the quartile cut-points used in constructing the Sydney Walkability Index to evaluate their appropriateness and the applicability of our findings to their geographic context. Further, modelling walkability as an index means we are unable to identify which built environment components in the index contribute to the observed associations with prevalence and geographic distribution of overweight and obesity, which would be useful for framing policy interventions. This was partly a choice for consistency with the expressed interest in the literature about the potential for "walkability indexes" to benchmark, inform and monitor development plans, but also because the added complexity would have made our models intractable. Our analysis used a two stage approach in which individual-level conditional probabilities of overweight and obesity were modelled first and then used as offset terms to adjust spatial models. While this approach is not uncommon in the epidemiological literature (see [39,42,122-124]), ideally we would have modelled individual and area-level effects simultaneously in a single, parsimonious model. However, despite the relative ease with which Besag, York and Mollié models can be fit in available software [75,178], they remain computationally prohibitive to implement outside of high performance computing environments when extended to multi-level problems comprising samples of the size used in our study [179]. Our units of analysis comprised Australian postal areas, which correspond in spatial extent to the upper limit of 
buffers sizes used in individual-level research linking walkability to high body mass but may not be representative of all spatial extents at which health and urban planning decisions occur. Finally, our study precludes causal inference due to its cross-sectional design.

\section{Conclusions}

Walkability indexes have been identified as potentially useful tools for planning and monitoring the built environment to improve health [27]. Our results provide support for their potential application to body mass outcomes by demonstrating that: (1) rates of overweight and obesity are negatively associated walkability at the postcode level for Sydney residents aged $\geq 45$ years; and (2) that area-level walkability makes a small but meaningful contribution to the geographic clustering of high body mass in the Sydney metropolitan region. Our results also suggest the presence of other unobserved and spatially structured factors contributing to this clustering. The Greater Sydney Region Plan aims to create healthy, resilient and socially connected communities over the next 40 years by creating fine scaled urban form, mixed land use and amenity within walkable urban centres [25]. The methods and outcomes described here may assist in the geographical targeting of strategies and monitoring their progress towards achieving its liveability objectives.

Author Contributions: Conceptualization, D.J.M., G.G.M., B.B.J. and A.E.B.; Data curation, D.J.M.; Formal analysis, D.J.M.; Methodology, D.J.M., G.G.M. and A.E.B.; Supervision, G.G.M. and A.E.B.; Writing-original draft, D.J.M., G.G.M., B.B.J. and A.E.B.; Writing—review \& editing, D.J.M., G.G.M., B.B.J. and A.E.B.

Acknowledgments: This research was completed using data collected through the 45 and Up Study (www.saxinstitute.org.au). The 45 and Up Study is managed by the Sax Institute in collaboration with major partner Cancer Council NSW; and partners: the National Heart Foundation of Australia (NSW Division); NSW Ministry of Health; NSW Government Family \& Community Services-Ageing, Carers and the Disability Council NSW; and the Australian Red Cross Blood Service. We thank the many thousands of people participating in the 45 and Up Study. Details on accessing 45 and Up Study data are available on the The Sax Institute website (www.saxinstitute.org.au/our-work/45-up-study). We wish to acknowledge Associate Professor Philayrath Phongsavan for her tireless and effective coordination of this project. DJM is grateful to Hevan Corrimal and WHC Sutherland for their ongoing support and providing office space. This study is part of the "Understanding the impact of the social, economic and environmental factors on the health of Australians in mid - later life; where are the opportunities for prevention?" study (National Health \& Medical Research Council (NHMRC) Grant 402810).

Conflicts of Interest: The authors declare no conflict of interest. This manuscript was reviewed for technical accuracy by The 45 and Up Study coordinating centre prior to its submission for peer review. Neither the 45 and Up Study coordinating centre nor the NHMRC had a role in the design of the study; the collection, analysis, or interpretation of data; the writing of the manuscript; or in the decision to publish the results.

\section{References}

1. Gregg, E.W.; Shaw, J.E. Global Health Effects of Overweight and Obesity. N. Engl. J. Med. 2017, 377, 80-81. [CrossRef] [PubMed]

2. Gakidou, E.; Afshin, A.; Abajobir, A.A.; Abate, K.H.; Abbafati, C.; Abbas, K.M.; Abd-Allah, F.; Abdulle, A.M.; Abera, S.F.; Aboyans, V.; et al. Global, regional, and national comparative risk assessment of 84 behavioural, environmental and occupational, and metabolic risks or clusters of risks, 1990-2016: A systematic analysis for the Global Burden of Disease Study 2016. Lancet 2017, 390, 1345-1422. [CrossRef]

3. Must, A.; Spadano, J.; Coakley, E.H.; Field, A.E.; Colditz, G.; Dietz, W.H. The disease burden associated with overweight and obesity. JAMA 1999, 282, 1523-1529. [CrossRef] [PubMed]

4. The GBD Obesity Collaborators. Health Effects of Overweight and Obesity in 195 Countries over 25 Years. N. Engl. J. Med. 2017, 377, 13-27. [CrossRef]

5. Lehnert, T.; Sonntag, D.; Konnopka, A.; Riedel-Heller, S.; König, H.H. Economic costs of overweight and obesity. Best Pract. Res. Clin. Endocrinol. Metab. 2013, 27, 105-115. [CrossRef] [PubMed]

6. Speakman, J.R. Obesity: The Integrated Roles of Environment and Genetics. J. Nutr. 2004, 134, 2090S-2105S. [CrossRef] [PubMed]

7. Rutter, H.; Savona, N.; Glonti, K.; Bibby, J.; Cummins, S.; Finegood, D.T.; Greaves, F.; Harper, L.; Hawe, P.; Moore, L.; et al. The need for a complex systems model of evidence for public health. Lancet 2017, 390, 2602-2604. [CrossRef] 
8. Lakerveld, J.; Mackenbach, J.D.; Rutter, H.; Brug, J. Obesogenic environment and obesogenic behaviours. In Advanced Nutrition and Dietetics in Obesity; Hankey, C., Ed.; John Wiley \& Sons, Incorporated: Newark, NJ, USA, 2017; Chapter 3.7, pp. 132-137.

9. Saelens, B.E.; Sallis, J.F.; Frank, L.D. Environmental correlates of walking and cycling: Findings from the transportation, urban design, and planning literatures. Ann. Behav. Med. 2003, 25, 80-91. [CrossRef] [PubMed]

10. Rose, G. Sick individuals and sick populations. Int. J. Epidemiol. 2001, 30, 427-432. [CrossRef] [PubMed]

11. Giles-Corti, B.; Timperio, A.; Bull, F.; Pikora, T. Understanding physical activity environmental correlates: Increased specificity for ecological models. Exerc. Sport Sci. Rev. 2005, 33, 175-181. [CrossRef] [PubMed]

12. Saelens, B.E.; Handy, S.L. Built environment correlates of walking: A review. Med. Sci. Sports Exerc. 2008, 40, S550-S566. [CrossRef] [PubMed]

13. Townshend, T.; Lake, A. Obesogenic environments: Current evidence of the built and food environments. Perspect. Public Health 2017, 137, 38-44. [CrossRef] [PubMed]

14. Feng, J.; Glass, T.A.; Curriero, F.C.; Stewart, W.F.; Schwartz, B.S. The built environment and obesity: A systematic review of the epidemiologic evidence. Health Place 2010, 16, 175-190. [CrossRef] [PubMed]

15. Durand, C.P.; Andalib, M.; Dunton, G.F.; Wolch, J.; Pentz, M.A. A systematic review of built environment factors related to physical activity and obesity risk: Implications for smart growth urban planning. Obes. Rev. 2011, 12, e173-e182. [CrossRef] [PubMed]

16. Mackenbach, J.D.; Rutter, H.; Compernolle, S.; Glonti, K.; Oppert, J.M.; Charreire, H.; De Bourdeaudhuij, I.; Brug, J.; Nijpels, G.; Lakerveld, J. Obesogenic environments: A systematic review of the association between the physical environment and adult weight status, the SPOTLIGHT project. BMC Public Health 2014, 14, 233. [CrossRef] [PubMed]

17. Garfinkel-Castro, A.; Kim, K.; Hamidi, S.; Ewing, R. Obesity and the built environment at different urban scales: Examining the literature. Nutr. Rev. 2017, 75, 51-61. [CrossRef] [PubMed]

18. Ding, D.; Gebel, K. Built environment, physical activity, and obesity: What have we learned from reviewing the literature? Health Place 2012, 18, 100-105. [CrossRef] [PubMed]

19. Leslie, E.; Coffee, N.; Frank, L.; Owen, N.; Bauman, A.; Hugo, G. Walkability of local communities: Using geographic information systems to objectively assess relevant environmental attributes. Health Place 2007, 13, 111-122. [CrossRef] [PubMed]

20. Sallis, J.F.; Frank, L.D.; Saelens, B.E.; Kraft, M. Active transportation and physical activity: Opportunities for collaboration on transportation and public health research. Transp. Res. Part A Policy Pract. 2004, 38, 249-268, [CrossRef]

21. Hamer, M.; Chida, Y. Active commuting and cardiovascular risk: A meta-analytic review. Prev. Med. 2008, 46, 9-13, [CrossRef] [PubMed]

22. Bird, E.L.; Ige, J.O.; Pilkington, P.; Pinto, A.; Petrokofsky, C.; Burgess-Allen, J. Built and natural environment planning principles for promoting health: An umbrella review. BMC Public Health 2018, 18, 930. [CrossRef] [PubMed]

23. City Planning Department of Helsinki. Helsinki City Plan: Urban Plan—The New Helsinki City Plan, Vision 2050. Available online: https://www.hel.fi/hel2/ksv/julkaisut/yos_2013-23_en.pdf (accessed on 2 February 2019).

24. Salt Lake City Council. Pedestrian \& Bicycle Master Plan. Available online: https://www.slc.gov/ transportation/bike/pbmp/ (accessed on 2 February 2019).

25. GreaterSydneyCommission. Greater Sydney Region Plan: A Metropolis of Three Cities-Connecting People. Available online: https: / www.planning.nsw.gov.au/plans-for-your-area/a-metropolis-of-three-cities/ametropolis-of-three-cities (accessed on 17 September 2018).

26. Transport for London. Walking Action Plan: Making London the World'S Most Walkable City. Available online: http:/ / content.tfl.gov.uk/mts-walking-action-plan.pdf (accessed on 2 February 2019).

27. Grasser, G.; Van Dyck, D.; Titze, S.; Stronegger, W. Objectively measured walkability and active transport and weight-related outcomes in adults: A systematic review. Int. J. Public Health 2013, 58, 615-625. [CrossRef] [PubMed]

28. Giles-Corti, B.; Macaulay, G.; Middleton, N.; Boruff, B.; Bull, F.; Butterworth, I.; Badland, H.; Mavoa, S.; Roberts, R.; Christian, H. Developing a research and practice tool to measure walkability: A demonstration project. Health Promot. J. Aust. 2014, 25, 160-166. [CrossRef] [PubMed] 
29. Cervero, R.; Kockelman, K. Travel demand and the 3Ds: Density, diversity, and design. Transp. Res. D Transp. Environ. 1997, 2, 199-219. [CrossRef]

30. Ewing, R.; Schmid, T.; Killingsworth, R.; Zlot, A.; Raudenbush, S. Relationship between urban sprawl and physical activity, obesity, and morbidity. Am. J. Health Promot. 2003, 18, 47-57, [CrossRef] [PubMed]

31. Krizek, K.J. Operationalizing Neighborhood Accessibility for Land Use-Travel Behavior Research and Regional Modeling. J. Plan. Educ. Res. 2003, 22, 270-287. [CrossRef]

32. Lopez, R. Urban sprawl and risk for being overweight or obese. Am. J. Public Health 2004, 94, 1574-1579. [CrossRef] [PubMed]

33. Levine, J.; Inam, A.; Torng, G.W. A Choice-Based Rationale for Land Use and Transportation Alternatives: Evidence from Boston and Atlanta. J. Plan. Educ. Res. 2005, 24, 317-330. [CrossRef]

34. Lee, C.; Moudon, A.V. The 3Ds + R: Quantifying land use and urban form correlates of walking. Transp. Res. Transp Environ. 2006, 11, 204-215. [CrossRef]

35. Ross, N.A.; Tremblay, S.; Khan, S.; Crouse, D.; Tremblay, M.; Berthelot, J.M. Body mass index in urban Canada: Neighborhood and metropolitan area effects. Am. J. Public Health 2007, 97, 500-508. [CrossRef] [PubMed]

36. Carr, L.J.; Dunsiger, S.I.; Marcus, B.H. Validation of Walk Score for estimating access to walkable amenities. Br. J. Sports Med. 2011, 45, 1144-1148, doi:10.1136/bjsm.2009.069609. [CrossRef] [PubMed]

37. Zick, C.D.; Hanson, H.; Fan, J.X.; Smith, K.R.; Kowaleski-Jones, L.; Brown, B.B.; Yamada, I. Re-visiting the relationship between neighbourhood environment and BMI: An instrumental variables approach to correcting for residential selection bias. Int. J. Behav. Nutr. Phys. Act. 2013, 10, 27. [CrossRef] [PubMed]

38. Frank, L.D.; Sallis, J.F.; Saelens, B.E.; Leary, L.; Cain, K.; Conway, T.L.; Hess, P.M. The development of a walkability index: Application to the Neighborhood Quality of Life Study. Br. J. Sports Med. 2010, 44, 924-933. [CrossRef] [PubMed]

39. Mayne, D.J.; Morgan, G.G.; Jalaludin, B.B.; Bauman, A.E. Does Walkability Contribute to Geographic Variation in Psychosocial Distress? A Spatial Analysis of 91,142 Members of the 45 and Up Study in Sydney, Australia. Int. J. Environ. Res. Public Health 2018, 15, 275. [CrossRef] [PubMed]

40. Mayne, D.; Morgan, G.; Willmore, A.; Rose, N.; Jalaludin, B.; Bambrick, H.; Bauman, A. An objective index of walkability for research and planning in the Sydney metropolitan region of New South Wales, Australia: An ecological study. Int. J. Health Geogr. 2013, 12, 61. [CrossRef] [PubMed]

41. Frank, L.D.; Andresen, M.A.; Schmid, T.L. Obesity relationships with community design, physical activity, and time spent in cars. Am. J. Prev. Med. 2004, 27, 87-96. [CrossRef] [PubMed]

42. Mayne, D.J.; Morgan, G.G.; Jalaludin, B.B.; Bauman, A.E. The contribution of area-level walkability to geographic variation in physical activity: A spatial analysis of 95,837 participants from the 45 and Up Study living in Sydney, Australia. Popul. Health Metr. 2017, 15, 38. [CrossRef] [PubMed]

43. Sugiyama, T.; Cole, R.; Koohsari, M.J.; Kynn, M.; Sallis, J.F.; Owen, N. Associations of local-area walkability with disparities in residents' walking and car use. Prev. Med. 2019, 120, 126-130. [CrossRef] [PubMed]

44. Rydin, Y.; Bleahu, A.; Davies, M.; Dávila, J.D.; Friel, S.; De Grandis, G.; Groce, N.; Hallal, P.C.; Hamilton, I.; Howden-Chapman, P.; et al. Shaping cities for health: Complexity and the planning of urban environments in the 21st century. Lancet 2012, 379, 2079-2108. [CrossRef]

45. Merom, D.; Ding, D.; Corpuz, G.; Bauman, A. Walking in Sydney: Trends in prevalence by geographic areas using information from transport and health surveillance systems. J. Transp. Health 2015, 2, 350-359. [CrossRef]

46. Papas, M.A.; Alberg, A.J.; Ewing, R.; Helzlsouer, K.J.; Gary, T.L.; Klassen, A.C. The built environment and obesity. Epidemiol. Rev. 2007, 29, 129-143. [CrossRef] [PubMed]

47. James, P.; Berrigan, D.; Hart, J.E.; Aaron Hipp, J.; Hoehner, C.M.; Kerr, J.; Major, J.M.; Oka, M.; Laden, F. Effects of buffer size and shape on associations between the built environment and energy balance. Health Place 2014, 27, 162-170. [CrossRef] [PubMed]

48. Villanueva, K.; Knuiman, M.; Nathan, A.; Giles-Corti, B.; Christian, H.; Foster, S.; Bull, F. The impact of neighborhood walkability on walking: Does it differ across adult life stage and does neighborhood buffer size matter? Health Place 2014, 25, 43-46. [CrossRef] [PubMed]

49. Sato, M.; Du, J.; Inoue, Y. Rate of Physical Activity and Community Health: Evidence From U.S. Counties. J. Phys. Act. Health 2016, 13, 640-648. [CrossRef] [PubMed] 
50. Riley, M.W. Special Problems of Sociological Analysis. In Sociological Research: A Case Approach; Riley, M.W., Merton, R.K., Eds.; Harcourt, Brace, and World: New York, NY, USA, 1963; Volume 1, pp. 700-725.

51. Alker H.A.J. A Typology of Ecological Fallacies. In Quantitative Ecological Analysis; Dogan, M., Rokkan, S., Eds.; Massachusetts Institute of Technology Press: Cambridge, MA, USA, 1969; pp. 69-86.

52. Schwartz, S. The fallacy of the ecological fallacy: The potential misuse of a concept and the consequences. Am. J. Public Health 1994, 84, 819-824. [CrossRef] [PubMed]

53. Ewing, R.; Hamidi, S. Measuring Sprawl 2014; Smart Growth America: Washington, DC, USA, 2014.

54. Hooper, P.; Knuiman, M.; Foster, S.; Giles-Corti, B. The building blocks of a 'Liveable Neighbourhood': Identifying the key performance indicators for walking of an operational planning policy in Perth, Western Australia. Health Place 2015, 36, 173-183. [CrossRef] [PubMed]

55. Fazli, G.S.; Creatore, M.I.; Matheson, F.I.; Guilcher, S.; Kaufman-Shriqui, V.; Manson, H.; Johns, A.; Booth, G.L. Identifying mechanisms for facilitating knowledge to action strategies targeting the built environment. BMC Public Health 2017, 17, 1. [CrossRef] [PubMed]

56. Cho, S.H.; Chen, Z.; Eastwood, D.B.; Yen, S.T. The effects of urban sprawl on body mass index: Where people live does matter? Consum. Interest Ann. 2006, 52, 159-169.

57. Ewing, R.; Hamidi, S. Measuring Urban Sprawl and Validating Sprawl Measures; Metropolitan Research Centre: Salt Lake City, UT, USA, 2010.

58. Hamidi, S.; Ewing, R.; Preuss, I.; Dodds, A. Measuring Sprawl and Its Impacts: An Update. J. Plan. Educ. Res. 2015, 35, 35-50. [CrossRef]

59. Kinge, J.M.; Steingrímsdóttir, O.A.; Strand, B.H.; Kravdal, Ø. Can socioeconomic factors explain geographic variation in overweight in Norway? SSM Popul. Health 2016, 2, 333-340. [CrossRef] [PubMed]

60. Gehlert, S.; Sohmer, D.; Sacks, T.; Mininger, C.; McClintock, M.; Olopade, O. Targeting Health Disparities: A Model Linking Upstream Determinants To Downstream Interventions. Health Aff. (Millwood) 2008, 27, 339-349. [CrossRef] [PubMed]

61. Pattenden, S.; Casson, K.; Cook, S.; Dolk, H. Geographical variation in infant mortality, stillbirth and low birth weight in Northern Ireland, 1992-2002. J. Epidemiol. Commun. Health 2011, 65, 1159-1165. [CrossRef] [PubMed]

62. Congdon, P. Variations in obesity rates between US counties: Impacts of activity access, food environments, and settlement patterns. Int. J. Environ. Res. Public Health 2017, 14, 1023. [CrossRef] [PubMed]

63. Sichieri, R.; Coitinho, D.C.; Leão, M.M.; Recine, E.; Everhart, J.E. High temporal, geographic, and income variation in body mass index among adults in Brazil. Am. J. Public Health 1994, 84, 793-798. [CrossRef] [PubMed]

64. Willms, J.D.; Tremblay, M.S.; Katzmarzyk, P.T. Geographic and Demographic Variation in the Prevalence of Overweight Canadian Children. Obesity 2003, 11, 668-673. [CrossRef] [PubMed]

65. Ackerson, L.K.; Kawachi, I.; Barbeau, E.M.; Subramanian, S.V. Geography of underweight and overweight among women in India: A multilevel analysis of 3204 neighborhoods in 26 states. Econ. Hum. Biol. 2008, 6, 264-280. [CrossRef] [PubMed]

66. Lebel, A.; Pampalon, R.; Hamel, D.; Thériault, M. The geography of overweight in Québec: A multilevel perspective. Can. J. Public Health 2009, 100, 18-23. [PubMed]

67. Simen-Kapeu, A.; Khule, S.; Veugelers, P.J. Geographic Differences in Childhood Overweight, Physical Activity, Nutrition and Neighbourhood Facilities: Implications for Prevention. Can. J. Public Health 2010, 101, 128-132. [PubMed]

68. El Mouzan, M.; Al Herbish, A.; Al Salloum, A.; Al Omar, A.; Qurachi, M. Regional variation in prevalence of overweight and obesity in Saudi children and adolescents. Saudi J. Gastroenterol. 2012, 18, 129-132. [CrossRef] [PubMed]

69. Toft, U.; Vinding, A.L.; Larsen, F.B.; Hvidberg, M.F.; Robinson, K.M.; Glümer, C. The development in body mass index, overweight and obesity in three regions in Denmark. Eur. J. Public Health 2015, 25, 273-278. [CrossRef] [PubMed]

70. Ajayi, I.O.; Adebamowo, C.; Adami, H.O.; Dalal, S.; Diamond, M.B.; Bajunirwe, F.; Guwatudde, D.; Njelekela, M.; Nankya-Mutyoba, J.; Chiwanga, F.S.; et al. Urban-rural and geographic differences in overweight and obesity in four sub-Saharan African adult populations: A multi-country cross-sectional study. BMC Public Health 2016, 16, 1126. [CrossRef] [PubMed] 
71. Adachi-Mejia, A.M.; Lee, C.; Lee, C.; Carlos, H.A.; Saelens, B.E.; Berke, E.M.; Doescher, M.P. Geographic variation in the relationship between body mass index and the built environment. Prev. Med. 2017, 100, $33-40$. [CrossRef] [PubMed]

72. Torres-Roman, J.S.; Urrunaga-Pastor, D.; Avilez, J.L.; Helguero-Santin, L.M.; Malaga, G. Geographic differences in overweight and obesity prevalence in Peruvian children, 2010-2015. BMC Public Health 2018, 18, 353. [CrossRef] [PubMed]

73. Mayhew, S. A Dictionary of Geography; Oxford University Press: Oxford, UK, 2015.

74. Fischer, M.M.; Getis, A. Introduction. In Handbook of Applied Spatial Analysis: Software Tools, Methods and Applications; Fischer, M.M., Getis, A., Eds.; Springer: Berlin/Heidelberg, Germany, 2010; Chapters 1-24.

75. Lawson, A.; Browne, W.J.; Vidal Rodeiro, C.L. Disease Mapping with WinBUGS and MLwiN; Statistics in Practice; Wiley: Hoboken, NJ, USA, 2003.

76. Smedley, B.; Amaro, H. Advancing the Science and Practice of Place-Based Intervention. Am. J. Public Health 2016, 106, 197-197. [CrossRef]

77. Miranda, M.L.; Edwards, S.E.; Keating, M.H.; Paul, C.J. Making the Environmental Justice Grade: The Relative Burden of Air Pollution Exposure in the United States. Int. J. Environ. Res. Public Health 2011, 8, 1755. [CrossRef] [PubMed]

78. Jia, P.; Cheng, X.; Xue, H.; Wang, Y. Applications of geographic information systems (GIS) data and methods in obesity-related research. Obes. Rev. 2017, 18, 400-411. [CrossRef] [PubMed]

79. Gutiérrez-Fisac, J.L.; Rodríguez Artalejo, F.; Guallar-Castillon, P.; Banegas Banegas, J.R.; del Rey Calero, J. Determinants of geographical variations in body mass index (BMI) and obesity in Spain. Int. J. Obes. 1999, 23, 342-347. [CrossRef]

80. Ford, E.S.; Mokdad, A.H.; Giles, W.H.; Galuska, D.A.; Serdula, M.K. Geographic Variation in the Prevalence of Obesity, Diabetes, and Obesity-Related Behaviors. Obes. Res. 2005, 13, 118-122. [CrossRef] [PubMed]

81. Lathey, V.; Guhathakurta, S.; Aggarwal, R.M. The Impact of Subregional Variations in Urban Sprawl on the Prevalence of Obesity and Related Morbidity. J. Plan. Educ. Res. 2009, 29, 127-141. [CrossRef]

82. Schuurman, N.; Peters, P.A.; Oliver, L.N. Are Obesity and Physical Activity Clustered? A Spatial Analysis Linked to Residential Density. Obesity 2009, 17, 2202-2209. [CrossRef] [PubMed]

83. Pórisdóttir, I.E.; Kristjansson, A.L.; Sigfusdottir, I.D.; Allegrante, J.P. The landscape of overweight and obesity in Icelandic adolescents: Geographic variation in body-mass index between 2000 and 2009. J. Commun. Health 2012, 37, 234-241. [CrossRef] [PubMed]

84. Myers, C.A.; Slack, T.; Martin, C.K.; Broyles, S.T.; Heymsfield, S.B. Regional disparities in obesity prevalence in the United States: A spatial regime analysis. Obesity 2015, 23, 481-487. [CrossRef] [PubMed]

85. Dutton, D.J.; McLaren, L. How important are determinants of obesity measured at the individual level for explaining geographic variation in body mass index distributions? Observational evidence from Canada using Quantile Regression and Blinder-Oaxaca Decomposition. J. Epidemiol. Commun. Health 2016, 70, 367-373. [CrossRef] [PubMed]

86. Paquet, C.; Chaix, B.; Howard, N.; Coffee, N.; Adams, R.; Taylor, A.; Thomas, F.; Daniel, M. Geographic clustering of cardiometabolic risk factors in metropolitan centres in France and Australia. Int. J. Environ. Res. Public Health 2016, 13, 519. [CrossRef] [PubMed]

87. Alkerwi, A.; Bahi, I.E.; Stranges, S.; Beissel, J.; Delagardelle, C.; Noppe, S.; Kandala, N.B. Geographic variations in cardiometabolic risk factors in Luxembourg. Int. J. Environ. Res. Public Health 2017, 14, 648. [CrossRef] [PubMed]

88. Smurthwaite, K.; Bagheri, N. Using Geographical Convergence of Obesity, Cardiovascular Disease, and Type 2 Diabetes at the Neighborhood Level to Inform Policy and Practice. Prev. Chronic Dis. 2017, 14, E91-E91. [CrossRef] [PubMed]

89. Samouda, H.; Ruiz-Castell, M.; Bocquet, V.; Kuemmerle, A.; Chioti, A.; Dadoun, F.; Kandala, N.B.; Stranges, S. Geographical variation of overweight, obesity and related risk factors: Findings from the European Health Examination Survey in Luxembourg, 2013-2015. PLoS ONE 2018, 13, e0197021. [CrossRef] [PubMed]

90. Australian Bureau of Statistics. Statistical Geography: Volume 1-Australian Standard Geographical Classification (ASGC), July 2006 (Catalogue No. 1216.0); Commonwealth of Australia: Canberra, Autralia, 2006.

91. Australian Bureau of Statistics. TableBuilder Basic. Available online: http://www.abs.gov.au/websitedbs / censushome.nsf/home/tablebuilder (accessed on 31 January 2018). 
92. Australian Bureau of Statistics. Statistical Geography: Volume 2-Census Geographic Areas, 2006 (Catalogue No. 2905.0); Commonwealth of Australia: Canberra, Australia, 2006.

93. 45 and Up Study Collaborators. Cohort profile: The 45 and Up Study. Int. J. Epidemiol. 2008, 37, 941-947. [CrossRef]

94. 45 and Up Study. Researcher Toolkit. Available online: https://www.saxinstitute.org.au/our-work/45-upstudy/for-researchers/ (accessed on 31 January 2018).

95. The 45 and Up Study. The 45 and Up Study Data Book-December 2011 Release. Available online: https:/ / www.saxinstitute.org.au/our-work/45-up-study/data-book/ (accessed on 31 January 2018).

96. Australian Bureau of Statistics. Socio-Economic Indexes for Areas (SEIFA)_Technical Paper, 2006; Australian Bureau of Statistics: Canberra, Australia, 2008.

97. WHO Expert Committee on Physical Status. Physical Status: The Use and Interpretation of Anthropometry. Report of a WHO Expert Committee; WHO Technical Report Series 854; World Health Organisation: Geneva, Switzerland, 1995.

98. Ng, S.P.; Korda, R.; Clements, M.; Latz, I.; Bauman, A.; Bambrick, H.; Liu, B.; Rogers, K.; Herbert, N.; Banks, E. Validity of self-reported height and weight and derived body mass index in middle-aged and elderly individuals in Australia. Aust. N. Z. J. Public Health 2011, 35, 557-563. [CrossRef] [PubMed]

99. Van Dyck, D.; Cerin, E.; Conway, T.L.; De Bourdeaudhuij, I.; Owen, N.; Kerr, J.; Cardon, G.; Frank, L.D.; Saelens, B.E.; Sallis, J.F. Perceived neighborhood environmental attributes associated with adults' transport-related walking and cycling: Findings from the USA, Australia and Belgium. Int. J. Behav. Nutr. Phys. Act. 2012, 9, 70. [CrossRef] [PubMed]

100. Van Dyck, D.; Cerin, E.; Conway, T.L.; De Bourdeaudhuij, I.; Owen, N.; Kerr, J.; Cardon, G.; Frank, L.D.; Saelens, B.E.; Sallis, J.F. Perceived neighborhood environmental attributes associated with adults' leisure-time physical activity: Findings from Belgium, Australia and the USA. Health Place 2013, 19, 59-68. [CrossRef] [PubMed]

101. Sallis, J.F.; Cerin, E.; Conway, T.L.; Adams, M.A.; Frank, L.D.; Pratt, M.; Salvo, D.; Schipperijn, J.; Smith, G.; Cain, K.L. Physical activity in relation to urban environments in 14 cities worldwide: A cross-sectional study. Lancet 2016, 387, 2207-2217. [CrossRef]

102. Astell-Burt, T.; Feng, X.; Kolt, G.S. Greener neighborhoods, slimmer people? Evidence from 246,920 Australians. Int. J. Obes. 2014, 38, 156, doi:10.1038/ijo.2013.64. [CrossRef] [PubMed]

103. Buchmueller, T.C.; Johar, M. Obesity and health expenditures: Evidence from Australia. Econ. Hum. Biol. 2015, 17, 42-58, doi:10.1016/j.ehb.2015.01.001. [CrossRef] [PubMed]

104. Charlton, K.; Kowal, P.; Soriano, M.; Williams, S.; Banks, E.; Vo, K.; Byles, J. Fruit and Vegetable Intake and Body Mass Index in a Large Sample of Middle-Aged Australian Men and Women. Nutrients 2014, 6, 2305. [CrossRef] [PubMed]

105. Creatore, M.I.; Glazier, R.H.; Moineddin, R.; Fazli, G.S.; Johns, A.; Gozdyra, P.; Matheson, F.I.; Kaufman-Shriqui, V.; Rosella, L.C.; Manuel, D.G. et al. Association of neighborhood walkability with change in overweight, obesity, and diabetes. JAMA 2016, 315, 2211-2220. [CrossRef] [PubMed]

106. Joshy, G.; Korda, R.J.; Attia, J.; Liu, B.; Bauman, A.E.; Banks, E. Body mass index and incident hospitalisation for cardiovascular disease in 158,546 participants from the 45 and Up Study. Int. J. Obes. 2014, $38,848$. [CrossRef] [PubMed]

107. Joshy, G.; Korda, R.J.; Bauman, A.; Van Der Ploeg, H.P.; Chey, T.; Banks, E. Investigation of Methodological Factors Potentially Underlying the Apparently Paradoxical Findings on Body Mass Index and All-Cause Mortality. PLoS ONE 2014, 9, e88641. [CrossRef] [PubMed]

108. Korda, R.J.; Liu, B.; Clements, M.S.; Bauman, A.E.; Jorm, L.R.; Bambrick, H.J.; Banks, E. Prospective cohort study of body mass index and the risk of hospitalisation: Findings from 246,361 participants in the 45 and Up Study. Int. J. Obes. 2013, 37, 790. [CrossRef] [PubMed]

109. Korda, R.J.; Joshy, G.; Paige, E.; Butler, J.R.G.; Jorm, L.R.; Liu, B.; Bauman, A.E.; Banks, E. The Relationship between Body Mass Index and Hospitalisation Rates, Days in Hospital and Costs: Findings from a Large Prospective Linked Data Study. PLoS ONE 2015, 10, e0118599. [CrossRef] [PubMed]

110. Magee, C.A.; Caputi, P.; Iverson, D.C. Is Sleep Duration Associated With Obesity in Older Australian Adults? J. Aging Health 2010, 22, 1235-1255. [CrossRef] [PubMed]

111. Magee, C.A.; Iverson, D.C.; Caputi, P. Sleep Duration and Obesity in Middle-aged Australian Adults. Obesity 2010, 18, 420-421. [CrossRef] [PubMed] 
112. Nguyen, B.; Bauman, A.; Ding, D. Incident Type 2 Diabetes in a Large Australian Cohort Study: Does Physical Activity or Sitting Time Alter the Risk Associated With Body Mass Index? J. Phys. Act. Health 2017, 14, 13-19. [CrossRef] [PubMed]

113. Pedisic, Z.; Grunseit, A.; Ding, D.; Chau, J.Y.; Banks, E.; Stamatakis, E.; Jalaludin, B.B.; Bauman, A.E. High sitting time or obesity: Which came first? Bidirectional association in a longitudinal study of 32,787 Australian adults. Obesity 2014, 22, 2126-2130. [CrossRef] [PubMed]

114. Kessler, R.C.; Andrews, G.; Colpe, L.J.; Hiripi, E.; Mroczek, D.K.; Normand, S.L.T.; Walters, E.E.; Zaslavsky, A.M. Short screening scales to monitor population prevalences and trends in non-specific psychological distress. Psychol. Med. 2002, 32, 959-976. [CrossRef] [PubMed]

115. Australian Bureau of Statistics. Use of the Kessler Psychological Distress Scale in ABS Health Surveys, Australia, 2007-08 (Catalgue No. 4817.0.55.001). Available online: http:/ /www.abs.gov.au/ausstats/abs@ .nsf/mf/4817.0.55.001 (accessed on 31 January 2018).

116. Australian Bureau of Statistics. National Survey of Mental Health and Wellbeing: Summary of Results; Commonwealth of Australia: Canberra, Australia, 2007.

117. Australian Bureau of Statistics. Australian Health Survey: Users' Guide, 2011-13 (Catalogue No. 4363.0.55.001). Available online: http:/ / www.abs.gov.au/ausstats/abs@.nsf/mf/4363.0.55.001 (accessed on 31 January 2018).

118. Ware, J. E., J.; Sherbourne, C.D. The MOS 36-item short-form health survey (SF-36). I. Conceptual framework and item selection. Med. Care 1992, 30, 473-483. [CrossRef] [PubMed]

119. Ware, J.E.; Snow, K.K.; Kosinski, M.; Gandek, B. SF-36 Health Survey: Manual and Interpretation Guide; The Health Institute, New England Medical Center: Boston, MA, USA, 1993.

120. Banks, E.; Jorm, L.; Rogers, K.; Clements, M.; Bauman, A. Screen-time, obesity, ageing and disability: Findings from 91,266 participants in the 45 and Up Study. Public Health Nutr. 2011, 14, 34-43. [CrossRef] [PubMed]

121. Waller, L.; Carlin, B. Disease Mapping. In Handbook of Spatial Statistics; Gelfand, A.E., Diggle, P.J., Feuentes, M., Guttorp, P., Eds.; Chapman \& Hall/CRC Handbooks of Modern Statistical Methods; CRC Press: Boca Raton, FL, USA, 2010; pp. 217-244.

122. Leroux, B.G.; Lei, X.; Breslow, N. Estimation of Disease Rates in Small Areas: A new Mixed Model for Spatial Dependence. In Statistical Models in Epidemiology, The Environment, and Clinical Trials; Halloran, M.E., Berry, D., Eds.; Springer: New York, NY, USA, 2000; pp. 179-191.

123. Klassen, A.C.; Kulldorff, M.; Curriero, F. Geographical clustering of prostate cancer grade and stage at diagnosis, before and after adjustment for risk factors. Int. J. Health Geogr. 2005, 4, 1. [CrossRef] [PubMed]

124. Waldhoer, T.; Wald, M.; Heinzl, H. Analysis of the spatial distribution of infant mortality by cause of death in Austria in 1984 to 2006. Int. J. Health Geogr. 2008, 7, 21. [CrossRef] [PubMed]

125. Besag, J.; York, J.; Mollié, A. Bayesian image restoration, with two applications in spatial statistics. Ann. Inst. Stat. Math. 1991, 43, 1-20. [CrossRef]

126. Cowles, M.K.; Carlin, B.P. Markov Chain Monte Carlo Convergence Diagnostics: A Comparative Review. J. Am. Stat. Assoc. 1996, 91, 883-904. [CrossRef]

127. Spiegelhalter, D.J.; Best, N.G.; Carlin, B.P.; Van Der Linde, A. Bayesian measures of model complexity and fit. J. R. Stat. Soc. Ser. B Stat. Methodol. 2002, 64, 583-639. [CrossRef]

128. Cramb, S.M.; Mengersen, K.L.; Baade, P.D. Developing the atlas of cancer in Queensland: Methodological issues. Int. J. Health Geogr. 2011, 10, 9. [CrossRef] [PubMed]

129. Holowaty, E.J.; Norwood, T.A.; Wanigaratne, S.; Abellan, J.J.; Beale, L. Feasibility and utility of mapping disease risk at the neighbourhood level within a Canadian public health unit: An ecological study. Int. J. Health Geogr. 2010, 9, 21. [CrossRef] [PubMed]

130. Mealing, N.M.; Banks, E.; Jorm, L.R.; Steel, D.G.; Clements, M.S.; Rogers, K.D. Investigation of relative risk estimates from studies of the same population with contrasting response rates and designs. BMC Med. Res. Methodol. 2010, 10, 1-12. [CrossRef] [PubMed]

131. Mayne, D.J.; Morgan, G.G.; Jalaludin, B.B.; Bauman, A.E. Is it worth the weight? Adjusting physical activity ratio estimates for individual-level non-response is not required in area-level spatial analyses of the 45 and Up Study cohort. In Proceedings of the 45 and Up Study Annual Forum, Sydney, Australia, 24 October 2017.

132. The 45 and Up Study. The 45 and Up Study Data Book-April 2010 Release. Available online: https: / / www.saxinstitute.org.au/our-work/45-up-study/data-book/ (accessed on 31 January 2018). 
133. Wasfi, R.A.; Dasgupta, K.; Orpana, H.; Ross, N.A. Neighborhood Walkability and Body Mass Index Trajectories: Longitudinal Study of Canadians. Am. J. Public Health 2016, 106, 934-940. [CrossRef] [PubMed]

134. James, P.; Kioumourtzoglou, M.A.; Hart, J.E.; Banay, R.F.; Kloog, I.; Laden, F. Interrelationships Between Walkability, Air Pollution, Greenness, and Body Mass Index. Epidemiololgy 2017, 28, 780-788. [CrossRef] [PubMed]

135. Kowaleski-Jones, L.; Brown, B.B.; Fan, J.X.; Hanson, H.A.; Smith, K.R.; Zick, C.D. The joint effects of family risk of obesity and neighborhood environment on obesity among women. Soc. Sci. Med. 2017, 195, 17-24. [CrossRef] [PubMed]

136. Loo, C.K.J.; Greiver, M.; Aliarzadeh, B.; Lewis, D. Association between neighbourhood walkability and metabolic risk factors influenced by physical activity: A cross-sectional study of adults in Toronto, Canada. BMJ Open 2017, 7. [CrossRef] [PubMed]

137. Méline, J.; Chaix, B.; Pannier, B.; Ogedegbe, G.; Trasande, L.; Athens, J.; Duncan, D.T. Neighborhood walk score and selected Cardiometabolic factors in the French RECORD cohort study. BMC Public Health 2017, 17, 960. [CrossRef] [PubMed]

138. Australian Urban Research Infrastructure Network (AURIN). Walkability Tools. Available online: https: / / docs.aurin.org.au/portal-help/analysing-your-data/walkability-tools / (accessed on 6 February 2019).

139. Frank, L.D.; Kerr, J.; Sallis, J.F.; Miles, R.; Chapman, J. A hierarchy of sociodemographic and environmental correlates of walking and obesity. Prev. Med. 2008, 47, 172-178. [CrossRef] [PubMed]

140. Rutt, C.D.; Coleman, K.J. Examining the relationships among built environment, physical activity, and body mass index in El Paso, TX. Prev. Med. 2005, 40, 831-841. [CrossRef] [PubMed]

141. Lunn, D.; Jackson, C.; Best, N.; Thomas, A.; Spiegelhalter, D. The BUGS Book: A Practical Introduction to Bayesian Analysis; Texts in Statistical Science; CRC Press: Boca Raton, FL, USA, 2012.

142. Latouche, A.; Guihenneuc-Jouyaux, C.; Girard, C.; Hémon, D. Robustness of the BYM model in absence of spatial variation in the residuals. Int. J. Health Geogr. 2007, 6, 1-8. [CrossRef] [PubMed]

143. Fotheringham, A.S.; Brunsdon, C.; Charlton, M. Quantitative Geography: Perspectives on Apatial Data Analysis; Sage Publications: London, UK, 2000; p. xii, 270p.

144. Fitzpatrick, J.; Griffiths, C.; Goldblatt, P. Introduction to the Volume. In Geographic Variations in Health; Griffiths, C., Fitzpatrick, J., Eds.; Office for National Statistics: London, UK, 2001.

145. Fontaine, K.R.; Cheskin, L.J.; Barofsky, I. Health-related quality of life in obese persons seeking treatment. J. Fam. Pract. 1996, 43, 265-270. [PubMed]

146. Katz, D.A.; McHorney, C.A.; Atkinson, R.L. Impact of obesity on health-related quality of life in patients with chronic illness. J. Gen. Intern. Med. 2000, 15, 789-796. [CrossRef] [PubMed]

147. Jia, H.; Lubetkin, E.I. The impact of obesity on health-related quality-of-life in the general adult US population. J. Public Health 2005, 27, 156-164. [CrossRef] [PubMed]

148. Frühbeck, G.; Toplak, H.; Woodward, E.; Yumuk, V.; Maislos, M.; Oppert, J.M. Obesity: The Gateway to Ill Health-An EASO Position Statement on a Rising Public Health, Clinical and Scientific Challenge in Europe. Obes. Facts 2013, 6, 117-120. [CrossRef] [PubMed]

149. Booth, H.P.; Prevost, A.T.; Gulliford, M.C. Impact of body mass index on prevalence of multimorbidity in primary care: Cohort study. Fam. Pract. 2014, 31, 38-43. [CrossRef] [PubMed]

150. Jackson, C.A.; Dobson, A.; Tooth, L.; Mishra, G.D. Body mass index and socioeconomic position are associated with 9-year trajectories of multimorbidity: A population-based study. Prev. Med. 2015, 81, 92-98. [CrossRef] [PubMed]

151. Kivimäki, M.; Kuosma, E.; Ferrie, J.E.; Luukkonen, R.; Nyberg, S.T.; Alfredsson, L.; Batty, G.D.; Brunner, E.J.; Fransson, E.; Goldberg, M.; et al. Overweight, obesity, and risk of cardiometabolic multimorbidity: Pooled analysis of individual-level data for 120813 adults from 16 cohort studies from the USA and Europe. Lancet Public Health 2017, 2, e277-e285. [CrossRef]

152. Katikireddi, S.V.; Skivington, K.; Leyland, A.H.; Hunt, K.; Mercer, S.W. The contribution of risk factors to socioeconomic inequalities in multimorbidity across the lifecourse: A longitudinal analysis of the Twenty-07 cohort. BMC Med. 2017, 15, 152. [CrossRef] [PubMed]

153. Stafford, M.; Hemingway, H.; Marmot, M. Current obesity, steady weight change and weight fluctuation as predictors of physical functioning in middle aged office workers: The Whitehall II study. Int. J. Obes. 1998, 22, 23-31. [CrossRef] 
154. Dowd, J.B.; Zajacova, A. Long-term obesity and physical functioning in older Americans. Int. J. Obes. 2015, 39, 502-507. [CrossRef] [PubMed]

155. Academy of Medical Sciences. Multimorbidity: A Priority for Global Health Research; Academy of Medical Sciences: London, UK, 2018.

156. Byles, J.E.; Gallienne, L.; Blyth, F.M.; Banks, E. Relationship of age and gender to the prevalence and correlates of psychological distress in later life. Int. Psychogeriatr. 2012, 24, 1009-1018. [CrossRef] [PubMed]

157. Byles, J.E.; Robinson, I.; Banks, E.; Gibson, R.; Leigh, L.; Rodgers, B.; Curryer, C.; Jorm, L. Psychological distress and comorbid physical conditions: Disease or disability? Depress Anxiety 2014, 31, 524-532. [CrossRef] [PubMed]

158. Ormel, J.; Rijsdijk, F.V.; Sullivan, M.; van Sonderen, E.; Kempen, G.I.J.M. Temporal and Reciprocal Relationship Between IADL/ADL Disability and Depressive Symptoms in Late Life. J. Gerontol. B Psychol. Sci. Soc. Sci. 2002, 57, P338-P347. [CrossRef] [PubMed]

159. Gaddey, H.L.; Holder, K. Unintentional weight loss in older adults. Am. Fam. Physician 2014, 89, 718-22. [PubMed]

160. Andrews, G.; Slade, T. Interpreting scores on the Kessler Psychological Distress Scale (K10). Aust. N. Z. J. Public Health 2001, 25, 494-497. [CrossRef] [PubMed]

161. Criqui, M.H. Response bias and risk ratios in epidemiologic studies. Am. J. Epidemiol. 1979, 109, $394-399$. [CrossRef] [PubMed]

162. Nohr, E.A.; Frydenberg, M.; Henriksen, T.B.; Olsen, J. Does low participation in cohort studies induce bias? Epidemiology 2006, 17, 413-418. [CrossRef] [PubMed]

163. Centre for Epidemiology and Evidence. Adult Population Health Survey. Available online: http://www. health.nsw.gov.au/surveys/adult/Pages/default.aspx (accessed on 12 December 2018).

164. Department of Finance, Services and Innovation. NSW Open Data Policy. Available online: https: //www.digital.nsw.gov.au/policy/data-information/making-data-open/nsw-open-data-policy (accessed on 4 February 2019).

165. Plantinga, A.J.; Bernell, S. The association between urban sprawl and obesity: Is it a two-way street? J. Reg. Sci. 2007, 47, 857-879. [CrossRef]

166. Eid, J.; Overman, H.G.; Puga, D.; Turner, M.A. Fat city: Questioning the relationship between urban sprawl and obesity. J. Urban Econ. 2008, 63, 385-404. [CrossRef]

167. Mokhtarian, P.L.; Cao, X. Examining the impacts of residential self-selection on travel behavior: A focus on methodologies. Trans. Res. Part B Methodol. 2008, 42, 204-228. [CrossRef]

168. Cao, X.; Mokhtarian, P.L.; Handy, S.L. Examining the Impacts of Residential Self-Selection on Travel Behaviour: A Focus on Empirical Findings. Transp. Rev. 2009, 29, 359-395. [CrossRef]

169. Kowaleski-Jones, L.; Zick, C.; Smith, K.R.; Brown, B.; Hanson, H.; Fan, J. Walkable neighborhoods and obesity: Evaluating effects with a propensity score approach. SSM Popul. Health 2018, 6, 9-15. [CrossRef] [PubMed]

170. Smith, K.R.; Zick, C.D.; Kowaleski-Jones, L.; Brown, B.B.; Fan, J.X.; Yamada, I. Effects of neighborhood walkability on healthy weight: Assessing selection and causal influences. Soc. Sci. Res. 2011, 40, 1445-1455. [CrossRef] [PubMed]

171. Smith, K.R.; Hanson, H.A.; Brown, B.B.; Zick, C.D.; Kowaleski-Jones, L.; Fan, J.X. Movers and stayers: How residential selection contributes to the association between female body mass index and neighborhood characteristics. Int. J. Obes. 2016, 40, 1384-1391. [CrossRef] [PubMed]

172. Hausman, J.A.; Abrevaya, J.; Scott-Morton, F.M. Misclassification of the dependent variable in a discrete-response setting. J. Econ. 1998, 87, 239-269. [CrossRef]

173. Tennekoon, V.; Rosenman, R. Systematically misclassified binary dependent variables. Commun. Stat. Theory Method. 2016, 45, 2538-2555. [CrossRef] [PubMed]

174. Openshaw, S.; Taylor, P.J. A Million or so Correlation Coefficients: Three Experiments on the Mmodifiable Areal Unit Problem. In Statistical Applications in the Spatial Sciences; Wrigley, N., Ed.; Pion: London, UK, 1979; pp. 127-144.

175. Openshaw, S. The Modifiable Areal Unit Problem; (CATMOG 38); Geo Books: Norwich, UK, 1984.

176. Greenland, S. Avoiding power loss associated with categorization and ordinal scores in dose-response and trend analysis. Epidemiology 1995, 6, 450-454. [CrossRef] [PubMed] 
177. Bennette, C.; Vickers, A. Against quantiles: Categorization of continuous variables in epidemiologic research, and its discontents. BMC Med. Res. Methodol. 2012, 12, 21. [CrossRef] [PubMed]

178. Goovaerts, P.; Gebreab, S. How does Poisson kriging compare to the popular BYM model for mapping disease risks? Int. J. Health Geogr. 2008, 7, 1-25. [CrossRef] [PubMed]

179. Huque, M.H.; Anderson, C.; Walton, R.; Ryan, L. Individual level covariate adjusted conditional autoregressive (indiCAR) model for disease mapping. Int. J. Health Geogr. 2016, 15, 25. [CrossRef] [PubMed]

(c) 2019 by the authors. Licensee MDPI, Basel, Switzerland. This article is an open access article distributed under the terms and conditions of the Creative Commons Attribution (CC BY) license (http://creativecommons.org/licenses/by/4.0/). 